\title{
Overexpression of FAM83A Promotes Proliferation and Metastasis Via Activating Wnt/ $\beta$-catenin Signaling Pathway in Head Neck Squamous Cell Carcinoma
}

Huan Ji

Nanjing Medical University https://orcid.org/0000-0002-9391-1166

Haiyang Song

Nanjing Medical University

Wei Zhang

Nanjing Medical University

Zeyu Wang

Nanjing Medical University

Pengfei Jiao

Nanjing Medical University

Yang Zheng

Nanjing Medical University

Yangyu Zheng

Nanjing Medical University

Yue Jiang

Nanjing Medical University

Jinping Zhang

Southern Medical University

Jiani Xu

Nanjing Medical University

Xuan Li

Nanjing Medical University

Hongming Du

Nanjing Medical University

Xu Ding

Nanjing Medical University

Heming Wu

Nanjing Medical University

Yi Zhong ( $D$ ohpzy@163.com )

Nanjing Medical University 


\section{Primary research}

Keywords: FAM83A, Head Neck Squamous Cell Carcinoma, Wnt/ $\beta$-catenin Signaling, Proliferation, Metastasis, Prognostic biomarker

Posted Date: November 12th, 2020

DOI: https://doi.org/10.21203/rs.3.rs-101880/v1

License: (c) (1) This work is licensed under a Creative Commons Attribution 4.0 International License. Read Full License 


\section{Abstract}

\section{Purpose:}

This research aimed to investigate FAM83A expression in head and neck squamous cell carcinoma (HNSCC) and its function in the proliferation and metastasis.

Materials and Methods:

FAM83A mRNA and protein expression in HNSCC was detected via TCGA database, primary HNSCC samples, and cell lines. The associations between FAM83A expression and clinicopathologic variables were evaluated through tissue microarrays. Besides, FAM83A knockdown and overexpression cell lines were constructed to assess its biological function including cell growth and metastasis in vitro and the relationship between FAM83A and epithelial-mesenchymal transition (EMT) was explored. Furthermore, two models of xenograft tumors in nude mice were used to assess the tumorigenicity and metastasis ability of FAM83A in vivo.

Results:

In the present study, we discovered the FAM83A expression was overexpressed in the HNSCC samples and was significantly associated with tumor size, lymph node status, and pathological grades.

Mechanically, FAM83A could promote HNSCC cell growth and metastasis by inducing EMT via activating Wnt/ $\beta$-catenin signaling pathway. Rescue experiment demonstrated the inhibition of $\beta$-catenin could counteract FAM83A function and there is a bi-directional signaling loop between FAM83A and Wnt/ $\beta$ catenin signaling pathways. Also, the FAM83A knockdown could suppress tumor growth and distant metastasis in HNSCC xenograft animal models.

\section{Conclusions:}

Taken together, we demonstrated that FAM83A is upregulated in HNSCC and it could promote cell growth and metastasis via activating Wnt/ $\beta$-catenin pathway in HNSCC, which may provide a novel therapeutic target in HNSCC treatment.

\section{Introduction}

In recent years, head and neck squamous cell carcinoma (HNSCC) is one of the most common cancers worldwide ,with more than 350,000 cancer-related deaths per year even though combined and multidisciplinary therapy has greatly advanced $[1,2]$. Therefore, more efforts should be made to explore the cellular and molecular mechanisms of HNSCC tumorigenesis. Thus, identification of new therapeutic targets for HNSCC is urgently needed for clinicians.

As the smallest member of the FAM83 family (FAM83A to $\mathrm{H}$ ), family with sequence similarity 83 , member A (FAM83A), is located on chromosome 8q24. In 2005, FAM83A, the conserved DUF1669 domain at its N- 
terminus, was firstly reported as a potential cancer biomarker involved in tumor progression[3]. Prior studies have suggested that FAM83A is upregulated in multiple human cancers and promotes the progression as an oncogenic gene in tumorigenesis[4-12]. For example, FAM83A expression is upregulated and promotes the progression of pancreatic cancer. FAM83A can contribute to HER2-positive cells growth and inhibits cell apoptosis in breast cancer. It is also reported FAM83A can be a diagnostic and prognostic marker of NSCLC and is closely related to tumor histology and signal transduction[13, 14]. FAM83A signaling can also stimulate epithelial-mesenchymal transition (EMT) by triggering the $\mathrm{PI} I \mathrm{~K} / \mathrm{AKT} /$ Snail pathway in NSCLC[15]. Also, FAM83A-S1 could promote the progression of lung cancer through the FAM83A/ERK pathway[16]. Recent studies have shown that FAM83A also interacts with Wnt and Hippo signaling pathway[17].

FAM83A can contribute to the growth of several malignant tumors. However, the function and the molecular mechanism of the FAM83A gene in HNSCC remains largely unknown. Here, we aim to clarify the role and mechanism of FAM83A in proliferation and metastasis of HNSCC cells, thus providing evidences for more potential therapeutic targets for HNSCC.

\section{Materials And Methods}

\subsection{Patients}

In brief, 242 patients including 148 males and 94 females, which underwent surgery and were diagnosed as primary HNSCC from 2009 to 2014 in the Affiliated Stomatological Hospital of Nanjing Medical University, were recruited. $242 \mathrm{HNSCC}$ samples including $12 \mathrm{HNSCC}$ adjacent normal tissues were used to make tissue microarrays[18]. The clinicopathologic information including age, gender, tumor size, lymph node status, histological grade, clinical stage and patients follow-up information were obtained from the patients' electronic medical records and follow-up visits. Besides, we collected 48 pairs fresh HNSCC tissues and adjacent normal tissues to extract mRNA and choose 10 pairs of them to examine FAM83A protein level. All experimental were in accordance with the Institutional Review Board of the Nanjing Medical University and complied with the Declaration of Helsinki (Approval ID 2019343).

\subsection{Immunohistochemistry and evaluation of immunoreactivity}

The procedure of immunohistochemistry (IHC) was carried out as we described previously[19], The stained slides were analyzed by two pathologists separately. FAM83A staining strength was grouped by combining intensity score (IS) and positive score (PS). IS was divided into four groups: negative (0), weak (1), moderate (2), and strong (3), whereas PS was categorized into four grades: negative (0), <10\% (1), $11 \%-50 \%$ (2), $51 \%-80 \%$ (3), and $>80 \%$ (4). We then calculated the immunoreactive score (IRS) by multiplying IS and PS. The final IRS were divided into two groups: low FAM83A expression ( $\leq 4)$, and high FAM83A expression (>4). The antibodies used in IHC was FAM83A (1:100; Proteintech, Rosemont, IL, USA).

\subsection{Cell lines and cell culture}


The human normal oral keratinocytes (HOK) and the human HNSCC cell lines including CAL27, FADU, HN4, HN6 SCC-9, and SCC-25 cell, were purchased from China Center for Type Culture Collection (Shanghai, China), CAL27 and HOK is cultured in DMEM medium (Gibco). FADU, HN4, and HN6 were cultured in DMEM/F12 medium (Gibco).

All culture medium contained $10 \%$ fetal bovine serum (FBS, Sciencell) and $1 \%$ penicillin/streptomycin. Cells were cultured in a humidified incubator containing $5 \% \mathrm{CO} 2$ at $37^{\circ} \mathrm{C}$.

\subsection{Lentiviral and siRNA transduction, and small-molecule inhibitor XAV-939}

The human FAM83A lentivirus targeting to upregulate FAM83A (LV-FAM83A: 5囚GGAGTGTGGAAGGAGAGAT-3区), the negative control (LV-NC), the shRNA lentivirus targeting to knock down FAM83A (shFAM83A: 5囚-GGAGUGUGGAAGGAGAGAUTT-3囚), and the shRNA negative control lentivirus (shNC) were ordered from GeneChem Co., Ltd (Shanghai, China). Small interfere RNA (siRNA) of $\beta$-catenin (si $\beta$-catenin: 5囚-GGACACAGCAGCAAUUUGUTT-3) was used to silence $\beta$-catenin expression. XAV-939 (Selleck, USA), a small-molecule inhibitor, resolved with DMSO, was used to suppress $\beta$-catenin expression.

\subsection{Real-time PCR and western blotting assays}

Real-time RT-PCR and western blotting assays were carried out in accordance with standard process as we described previously[19]. Nuclear and cytoplasmic protein extraction from cells is in accordance with the TRIZOL reagent (Invitrogen) manufacturer's protocol. The primers used as follows, FAM83A: forward 5'- ATCCGGAGTGTGGAAGGAGAG -3', reverse 5'- TCCAGACAGGACAAATCTCCAGT -3'; E-cadherin: forward 5'-GCCTTATGATTCTCTGCTCGTG -3', reverse 5'-GCCCCATTCGTTCAAGTAGTC-3'; N-cadherin: forward 5'GTGAGCCTGCAGATTTTAAGGTG-3', reverse 5'-GTTGGCTTCAGGCTCATTTTACT-3'; Vimentin: forward 5'CTGGATTCACTCCCTCTGGTT-3', reverse 5'-TCGTGATGCTGAGAAGTTTCGTT-3'; Snail: forward 5'TTCTCACTGCCATGGAATTCC-3', reverse 5'- GCAGAGGACACAGAACCAGAAA-3'; $\beta$-catenin: forward 5'TGACAAAACTGCTAAATGACGAGG-3', reverse 5'- CGCATGATAGCGTGTCTGGA-3'; C-myc: forward 5'CCACGAAACTTTGCCCATAG -3', reverse 5'- TGCAAGGAGAGCCTTTCAGAG-3';Cyclin D1: forward 5'TGTCCCACTCCTACGATACGC -3', reverse 5'- CAGCATCTCATAAACAGGTCACTAC-3'; GAPDH: forward 5'GACGTAGGGAGTGAAGGT C-3', reverse 5'-GAGAGTTCAGATGTTGATGG-3'. Primary antibodies were as follows: GAPDH (1:1000; Proteintech, Rosemont, IL, USA), Lamin B1 (1:1000; CST), FAM83A (1:1000; Proteintech, Rosemont, IL, USA), E-cadherin (1:1000; CST) $₫ N$-cadherin $₫ 1: 1000 ; C S T)$, Vimentin $(1: 1000$; CST), Snail (1:1000; Proteintech, Rosemont, IL, USA), $\beta$-catenin (1:1000; CST), C-myc (1:1000; CST), and Cyclin D1 (1:1000; CST).

\subsection{Cell migration and invasion assays}

For the migration assay, $8 \times 10^{4} \mathrm{HNSCC}$ cells in $200 \mathrm{ul}$ complete medium were seeded into the upper compartment of a transwell insert with $8 \mathrm{~mm}$ pores (Costar, Lowell, MA, USA). The lower chamber was filled with 700ul basal medium containing 10\% fetal bovine serum (FBS). After 12h (HN6 cell line) or $24 \mathrm{~h}$ 
(CAL27, FADU and HN4 cell lines), the invaded cells adhered to the lower compartment were fixed, stained, and counted under an inverted microscope (Olympus, Tokyo, Japan). For the invasion assay, $2 \times 10^{5}$ cells/well were plated and the upper compartment was pre-coated with Matrigel (Corning, Bedford, MA, USA).

\subsection{Cell viability measurement}

Cell viability was detected by CCK-8 assay (Dojindo Molecular Technologies, Kumamoto, Japan). Cells were seeded and cultured in 96-well plates at a density of 2000 cells/well. Every other day, CCK-8 reagent was added and the absorbance was determined at $450 \mathrm{~nm}$.

\subsection{Wound-healing assay}

Cells were seeded into six-well plates and scratched with a 10ul pipette tip when cells grew to $90 \%$ confluence, and then cells were incubated with complete medium. At 0,12 , or 24 hours, wound closure was photographed and its percentage was calculated.

\subsection{Immunofluorescence staining}

Cells were plated onto coverslips and fixed in 4\% paraformaldehyde for 20 mins when cells grew to $50 \%$ confluency. Then, the coverslips were permeabilized with or without $0.3 \%$ Triton, incubated with goat serum for 30 minutes and primary antibody overnight at $4{ }^{\circ} \mathrm{C}$. After the slides were incubated with DAPI (Life Technologies, USA), cells were observed and photographed under an FV1000 laser confocal scanning microscope (Tokyo, Japan). Primary antibodies used were as follows: E-cadherin (1:100, CST), Vimentin $(1: 100$, CST), and $\beta$-catenin $(1: 100 ;$ CST).

\subsection{In vivo assay}

All animal experiments were in accordance with the Animal Use and Care Committee of the Affiliated Hospital of Stomatology, Nanjing Medical University (IACUC-1906018). Female 4-6 weeks old BALB/C nude mice used were purchased from Vital River Laboratory Animal Technology Co.Ltd (Beijing, China). To explore the role of FAM83A on HNSCC tumor growth, a total of 2×106 FAM83A knockdown cells and control cells were injected into the right armpit of mice. Tumor growth and body weight were determined every three days. After 25 days, mice were killed and tumors were stripped carefully. To explore the role of FAM83A on HNSCC tumor metastatic potential, a total of $1 \times 105$ cells were injected into the nude mice by tail intravenous. After 40 days, mice lungs were dissected carefully and the number of metastatic nodes were counted.

\subsection{Statistical analysis}

Data were presented as the mean $\pm \mathrm{s}$.d from three independent experiments and all data were analyzed via GraphPad Prism 7 (San Diego, CA, USA) software. $P<0.05$ was considered significant in all experiments. 


\section{Results}

\subsection{FAM83A overexpression is associated with clinicopathological characteristics in HNSCC.}

To explore FAM83A role in HNSCC, we examined FAM83A expression through 3 tissue microarrays consisting of 242 human HNSCC samples and 12 adjacent normal tissues. Obviously, HNSCC tissues showed stronger cytoplasmic staining of FAM83A and adjacent normal tissues exhibited a lower or negative expression of FAM83A through IHC analysis (Fig.1A-C). We then analyzed the relationship between FAM83A expression and clinicopathological features (Table 1 and Figure 1D-G). Elevated FAM83A staining was detected in bigger tumor size and elevated FAM83A staining was detected in higher pathological stages (Figure1D and 1F). Besides, advanced HNSCC patients with lymph node metastasis had higher FAM83A expression levels than those in early stage patients with no lymph node metastasis (Figure1E). However, FAM83A expression has no correction with clinical stage (Figure1G). Above all, we found that increased FAM83A expression was related to tumor volumes, lymph node status, and pathological grade.

To further clarify the role of FAM83A in HNSCC, we collected 48 pairs freshly tumor tissues which were diagnosed as HNSCC and adjacent normal tissues. 48 pairs of tissues were used to extract mRNA and 10 pairs of tissues among them were used to extract protein. Our results from qRT-PCR in 48 HNSCC samples and paired normal tissues demonstrated that FAM83A mRNA was significantly increased in HNSCC than paired normal tissues (Fig.1H). Among 10 pairs of HNSCC samples and normal tissues, 8 pairs expressed a higher FAM83A protein level in tumor tissues (Fig.1J). These phenomenon illustrated FAM83A had a higher expression level in HNSCC samples than adjacent normal tissues both at mRNA and protein level. Besides, we evaluated FAM83A mRNA expression level through available TCGA database and found FAM83A is upregulated in 519 HNSCC samples compared to 44 normal tissues (Fig.11). Meanwhile, we assessed FAM83A expression level in HNSCC cell lines and human normal oral keratinocytes (HOK) and found that FAM83A expression in HNSCC cell lines was higher than that in HOK cells (Fig.1K).

\subsection{FAM83A promotes HNSCC cells growth and metastatic in vitro.}

To further explore FAM83A function in HNSCC, we constructed the FAM83A knockdown and overexpression cell lines and verify the efficiency of interference both at the mRNA and protein levels through qRT-PCR and Western blotting assays. CAL27 and FADU cells were transfected with specific shRNA lentivirus (Fig.2A and 2B), while HN4 and HN6 cells were transfected with FAM83A overexpression lentivirus (LV-FAM83A) (Fig.3A and 3B). We found that FAM83A knockdown did not change CAL27 and FADU cell morphology (Fig.2C), but FAM83A overexpression made HN4 and HN6 cells become longer and thinner (Fig.3C). Meanwhile, CCK8 assay found FAM83A downregulation impaired cell viability in CAL27 and FADU cells (Fig.2D), while, FAM83A upregulation promoted cell growth in HN4 and HN6 cells (Fig.3D). In the wound healing experiment, the migration of FAM83A knockdown group was significantly slower than the negative control group in CAL27 and FADU cell lines (Fig.2E-F). Conversely, FAM83A overexpression group enhanced the migratory ability in HN4 and HN6 cells lines (Fig.3E-F). Besides, 
transwell assays showed that the downregulation of FAM83A significantly weakened the migratory and invasive ability in CAL27 and FADU cell lines (Fig.2G-H) and FAM83A upregulation significantly enhanced the migratory and invasive ability in HN4 and HN6 cell lines (Fig.3G-H). These results indicated FAM83A promoted HNSCC cells growth and metastatic in vitro.

\subsection{FAM83A promotes EMTin HNSCC cell lines}

As we know, EMT plays an important role in tumor progression, so we investigated whether FAM83A promotes EMT progression. As shown in Figure 4A-4H, after FAM83A were knocked down in CAL27 and FADU cells, the epithelial marker E-cadherin descended and mesenchymal markers including $\mathrm{N}$-cadherin, Vimentin and Snail elevated both at protein and mRNA level (Fig.4A-C). As expected, FAM83A overexpression exerted the opposite effects in HN4 and HN6 cells (Fig.4D-E). Both Real-time PCR and western blotting analysis demonstrated this result. Furthermore, immunofluorescence staining assay confirmed FAM83A downregulation decreased Vimentin but increased E-cadherin expression in CAL27 cell lines, whereas the upregulation of FAM83A reverted this phenomenon in HN6 cell lines (Fig.4G, H). Taken together, FAM83A promoted EMT in HNSCC.

\subsection{FAM83A promotes nuclear $\beta$-catenin accumulation and suppresses degradation of cytosolic $\beta$-catenin in HNSCC cells.}

To investigate the possible mechanism of FAM83A in regulating the proliferation, migration, and invasion of HNSCC, we detected Wnt/ $\beta$-catenin signaling pathway downstream genes such as C-myc and Cyclin D1. Compared with the scramble group, the FAM83A knockdown group showed a downregulation of Cmyc and Cyclin D1. Meanwhile, FAM83A overexpression group cells showed a upregulation of C-myc and Cyclin D1 (Figure.4A-F). Western blotting analysis demonstrated nuclear $\beta$-catenin decreased while cytosolic $\beta$-catenin increased after FAM83A knockdown in CAL27 and HN6 cells. Meanwhile, $\beta$-catenin increased in the nucleus and decreased in the cytosolic when FAM83A overexpressed (Figure.5A and 5C). Besides, Immunofluorescence assay of $\beta$-catenin showed lower level of nuclear $\beta$-catenin in the FAM83A knockdown groups and higher level of nuclear $\beta$-catenin in the FAM83A-overexpressing groups in CAL27 and HN6 cells (Figure.5B and 5D). These phenomenon indicated that FAM83A activated the Wnt/ $\beta$ catenin signaling pathway. We treated HNSCC cells with 50umol XAV-939 which could suppress $\beta$-catenin expression and we detected cell viability, migration and invasion ability all decreased after treatment with XAV-939 in CAL27 and HN6 cells (Figure.5E-I). At the same time, western blotting assay found that Ecadherin expression was declined, $\mathrm{N}$-cadherin, Vimentin and Snail expression were increased when cells were treated with XAV-939 in CAL27 and HN6 FAM83A-overexpression group cells (Figure.5J). Meanwhile, the downregulation of $\beta$-catenin induced by XAV-939 decreased FAM83A protein expression (Figure.5J). This suggests that there may be a loop feedback between FAM83A and $\beta$-catenin. To further prove it, we treated CAL27 and HN6 cells with small interfering RNA (siRNA) of $\beta$-catenin for $48 \mathrm{~h}$, we also found $\beta$ catenin inactivation brought about FAM83A protein downregulation. Taken together, the above findings suggested that FAM83A promotes cell proliferation and metastasis in HNSCC by activating Wnt/ $\beta$ catenin signaling pathway and $\beta$-catenin also regulates FAM83A expression. 


\subsection{FAM83A promotes tumor growth and distant metastasis in vivo.}

To study FAM83A function on tumor growth in HNSCC, we used a subcutaneous xenograft tumor model and injected cells $(2 * 105)$ into the right flank of mice. Compared with the tumors formed by the negative control cells, the tumors separated from mice injected with the FAM83A-knockdown cells were smaller and showed a less tumor formation ability (Fig. 6A). The tumors formed in the negative control group possessed a larger tumor volume and weight than shFAM83A group during its development (Fig. 6B and 6C). HE staining assay demonstrated the tumors separated from nude mice were HNSCC (Fig. 6D). Besides, IHC assay demonstrated tumors dissected from negative control group had a stronger FAM83A staining when compared with the other group (Fig. 6E). Moreover, we used an experimental metastasis assay to explore its function on tumor metastasis potential and injected cells $(1 * 105)$ into the tail vein of nude mice. As described in Figure. 6F and 6G, mice injected with shFAM83A group cells formed less pulmonary metastases (an average of 3 nodules per lung) than shNC group (an average of 11 nodules per lung) 42 days later (Fig. 6F and 6G). Furthermore, HE staining assays demonstrated that the number of metastatic lesions significantly declined in shFAM83A group mice compared with shNC group (Fig. $6 \mathrm{H})$. These findings suggested that FAM83A promoted tumor growth and metastasis in HNSCC.

\section{Discussion}

Head and neck squamous cell carcinoma (HNSCC) is a non-skin malignant tumor with high mortality rate. The carcinogenesis of HNSCC is complex which involves coefficient function of multiple feedback loops, and cross-talk communications. Different genetic alterations interact with each other and will be taken into consideration to guide the targeted therapy in HNSCC. FAM83A, whose expression is upregulated in cancers, often functions as an oncogenic gene in tumorigenesis. However, the function of FAM83A in HNSCC had not been reported and particularly required further exploration.

In our study, the function and mechanism of FAM83A in HNSCC were profoundly investigated. First, Immunohistochemistry in human HNSCC microarrays showed aberrant overexpression of FAM83A in a large number of patients compared with adjacent normal tissues examined in our study. It is interesting to note that FAM83A positivity was observed as brown membranous and cytoplasmic immunostaining in HNSCC group, consistent with the previous report. Our results showed that increased FAM83A expression was positively correlated with advanced tumor size, lymph node metastasis, and advanced pathological stage respectively. The clinical specimens showed that FAM83A may play a key role in the migration and metastasis of HNSCC in the present study. Then, FAM83A expression was noticeably amplified in HNSCC tissues and cells by RT-qPCR and Western Blotting. This was consistent with the results of TCGA database indicating that HNSCC samples have a higher expression of FAM83A than that in adjacent tissues. Therefore, FAM83A might serve as an oncogene in HNSCC.

Furthermore, we constructed stably transfected FAM83A knockdown and overexpressed HNSCC cells and assessed the cell viability, migration and invasion in the stably transfected cells. HNSCC cells presented fibroblast-like phenotypes, exhibiting a more elongated and spindle-like shape after FAM83A 
overexpression. Depletion of FAM83A dramatically inhibited cell proliferation, migration, and invasion significantly in HNSCC cells. Moreover, we found that knockdown of FAM83A expression in HNSCC cells decreased metastatic foci in vivo. FAM83A overexpression exerted the opposite effects. All these results illustrated that the up-regulation of FAM83A could enhance cell proliferation, migration and invasion in HNSCC. Taken together, these data indicate that FAM83A plays an oncogenic role to promote HNSCC progression.

The epithelial to mesenchymal transition (EMT) program plays a vital role in cancer metastasis[20, 21]. The down-regulation of epithelium markers such as E-cadherin and the up-regulation of mesenchymal marker such as N-cadherin, Vimentin and Snail are the most common hallmarks of EMT[22]. Prior studies have indicated that initiation of EMT in tumor is frequently observed in head and neck squamous cell carcinoma metastasis, and EMT[23]. In our study, HNSCC cells presented fibroblast-like phenotypes, showing a more elongated and spindle-like shape in HNSCC cells after FAM83A overexpression. Also, Ecadherin was increased while N-cadherin, Vimentin, and Snail were inhibited after silencing FAM83A in CAL27 and FADU cell lines, which suggested that tumor progression and cell migration were inhibited, consistent with the previous study. All these data suggested that FAM83A can induce the activation of the EMT signaling pathway In HNSCC cells. Therefore, FAM83A might play a vital role in the EMT of HNSCC.

It is suggested that aberrant changes of Wnt/ $\beta$-catenin signaling are involved in EMT[24]. Wnt can activate $\beta$-catenin at a downstream location in the Wnt/ $\beta$-catenin signaling[25]. The $W n t / \beta$-catenin signaling pathway can play a key role in cell proliferation, differentiation, and regeneration[26]. It is interesting to note that FAM83A can interact with Wnt signaling pathway in lung cancers. Thus, we aimed to examine whether there is any connection between FAM83A and Wnt/ $\beta$-catenin signaling pathway in the present study. Thus we constructed FAM83A overexpression and knockdown cell lines and examined the relation between the expression of FAM83A and Wnt/ $\beta$-catenin signaling pathway. Here, we found the overexpression of FAM83A promoted the Wnt signaling pathway. In FAM83A-overexpressed cells, the activity of Wnt pathway was upregulated, and its downstream targets C-myc and Cyclin D1 were both upregulated. Besides, western blotting assays and immunofluorescence staining assays demonstrated $\beta$ catenin entered into nuclear from cytoplasmic in CAL27 and HN6 FAM83A-upregulated cells,demonstrating the Wnt-pathway-mediated $\beta$-catenin activation. These results were further demonstrated by using the $\beta$-catenin inhibitor to pharmacologically block the Wnt signaling pathway. In the rescue experiment, our experiments demonstrated that the inhibitor, XAV-939, could reverse the promoting effect of FAM83A in HNSCC. Furthermore,we found for the first time that $\beta$-catenin inhibitor could also downregulate the expression of FAM83A in CAL27 and HN6 cells. We finally proved this phenomenon with si- $\beta$-catenin transfection. In conclusion, the present study demonstrates bi-directional signaling loop between FAM83A and Wnt- $\beta$-catenin signaling pathway. The results provide evidence that the Wnt- $\beta$-catenin-mediated EMT and metastasis by FAM83A.

In our study, we presented evidence for the first time that overexpression of FAM83A in HNSCC tissues was positively correlated with advanced tumor size, lymph node metastasis, and advanced pathological stage. Besides, the present study is the first to reveal that FAM83A accelerates the EMT of HNSCC by 
facilitating WNT/ $\beta$-catenin and metastasis in HNSCC and there is a bi-directional signaling loop between FAM83A and Wnt- $\beta$-catenin signaling pathways. Our research provided a novel target for HNSCC for the early diagnosis and treatment in HNSCC.

\section{Declarations}

\section{Acknowledgements}

All authors are acknowledged for their contribution to the study.

\section{Authors' contributions}

Yi Zhong and Heming Wu designed this experiment. Huan Ji, Haiyang Song, Wei Zhang, Zeyu Wang, and Pengfei Jiao performed all procedures of experiments. Yang Zheng, Yangyu Zheng, Zhang Jinping, Yue Jiang, Jiani $\mathrm{Xu}$, Xuan Li, Hongming Du, and Xu Ding analyzed data. Yi Zhong and Heming Wu wrote the manuscript. All authors edited and approved the manuscript.

\section{Authors' information}

Not applicable.

\section{Funding}

The author(s) disclosed receipt of the following financial support for the research, authorship, and/or publication of this article: This study was supported by the Natural Science Foundation of Jiangsu Province (Grant No BK2018040793).

\section{Availability of data and materials}

The datasets generated and/or analyzed during the current study are not publicly available but are available from the corresponding author upon reasonable request.

\section{Ethics approval and consent to participate}

This study was approved by the ethical committee of the Nanjing Medical University. All cells donors signed informed consent documents. The authors declare that they have no conflict of interest.

\section{Consent for publication}

Not applicable

\section{Competing interests}

The authors have no competing interests to declare. 


\section{References}

1. Erratum: Global cancer statistics 2018: GLOBOCAN estimates of incidence and mortality worldwide for 36 cancers in 185 countries. CA Cancer J Clin 2020, 70(4):313.

2. Zhang XM, Song LJ, Shen J, Yue H, Han YQ, Yang CL, Liu SY, Deng JW, Jiang Y, Fu GH et al: Prognostic and predictive values of immune infiltrate in patients with head and neck squamous cell carcinoma.HUM PATHOL 2018, 82:104-112.

3. Li Y, Dong X, Yin Y, Su Y, Xu Q, Zhang Y, Pang X, Zhang Y, Chen W: BJ-TSA-9, a Novel Human TumorSpecific Gene, Has Potential as a Biomarker of Lung Cancer. NEOPLASIA 2005, 7(12):1073-1080.

4. JH Mao AM, Lee SY, Hang B, Hao W, Bissell MJ, Mao JH: FAM83 family oncogenes are broadly involved in human cancers: an integrative multi-omics approach. MOL ONCOL 2017, 11(2):167-179.

5. Bartel CA, Jackson MW: HER2-positive breast cancer cells expressing elevated FAM83A are sensitive to FAM83A loss. PLOS ONE 2017, 12(5):e176778.

6. Chen S, Huang J, Liu Z, Liang Q, Zhang N, Jin Y: FAM83A is amplified and promotes cancer stem cell-like traits and chemoresistance in pancreatic cancer. ONCOGENESIS 2017, 6(3):e300.

7. Cipriano R, Miskimen KLS, Bryson BL, Foy CR, Bartel CA, Jackson MW: Conserved Oncogenic Behavior of the FAM83 Family Regulates MAPK Signaling in Human Cancer. MOL CANCER RES 2014, 12(8):1156-1165.

8. Grant S: FAM83A and FAM83B: candidate oncogenes and TKI resistance mediators. J CLIN INVEST 2012, 122(9):3048-3051.

9. Lee S, Meier R, Furuta S, Lenburg ME, Kenny PA, Xu R, Bissell MJ: FAM83A confers EGFR-TKI resistance in breast cancer cells and in mice. J CLIN INVEST 2012, 122(9):3211-3220.

10. Liu L, Liao G, He P, Zhu H, Liu P, Qu Y, Song X, Xu Q, Gao Q, Zhang Y et al: Detection of circulating cancer cells in lung cancer patients with a panel of marker genes. BIOCHEM BIOPH RES CO 2008, 372(4):756-760.

11. Liu L, Ma C, Xu Q, Cheng L, Xiao L, Xu D, Gao Y, Wang J, Song H: A rapid nested polymerase chain reaction method to detect circulating cancer cells in breast cancer patients using multiple marker genes.ONCOL LETT 2014, 7(6):2192-2198.

12. Xu J, Lu W: FAM83A exerts tumorsuppressive roles in cervical cancer by regulating integrins. INT J ONCOL 2020, 57(2):509-521.

13. Richtmann S, Wilkens D, Warth A, Lasitschka F, Winter H, Christopoulos P, Herth FJF, Muley T, Meister M, Schneider MA: FAM83A and FAM83B as Prognostic Biomarkers and Potential New Therapeutic Targets in NSCLC. CANCERS 2019, 11(5):652.

14. Zhang J, Lin Y, Xiao B, Yu B: Overexpression of Family with Sequence Similarity 83, Member A (FAM83A) Predicts Poor Clinical Outcomes in Lung Adenocarcinoma. MED SCI MONITOR 2019, 25:4264-4272.

15. Zhou F, Geng J, Xu S, Meng Q, Chen K, Liu F, Yang F, Pan B, Yu Y: FAM83A signaling induces epithelial-mesenchymal transition by the PI3K/AKT/Snail pathway in NSCLC. Aging 2019, 11(16). 
16. He J, Yu J: Long noncoding RNA FAM83A-AS1 facilitates hepatocellular carcinoma progression by binding with NOP58 to enhance the mRNA stability of FAM83A. BIOSCIENCE REP 2019, 39(11).

17. Zheng Y, Li Z, Lei L, Liu C, Wang Z, Fei L, Yang M, Huang W, Xu H: FAM83A Promotes Lung Cancer Progression by Regulating the Wnt and Hippo Signaling Pathways and Indicates Poor Prognosis. FRONT ONCOL 2020, 10.

18. Zheng Y, Wang C, Song A, Jiang F, Zhou J, Li G, Zhang W, Ye J, Ding X, Zhang W et al: CMTM6 promotes cell proliferation and invasion in oral squamous cell carcinoma by interacting with NRP1. AM J CANCER RES 2020, 10(6):1691-1709.

19. Ji H, Ding X, Zhang W, Zheng Y, Du H, Zheng Y, Song H, Li M, Jiang Y, Xie J et al: Claudin-7 Inhibits Proliferation and Metastasis in Salivary Adenoid Cystic Carcinoma Through Wnt/ $\beta$-Catenin Signaling. CELL TRANSPLANT 2020, 29:1504093290.

20. Lambert AW, Pattabiraman DR, Weinberg RA: Emerging Biological Principles of Metastasis. CELL 2017, 168(4):670-691.

21. Pastushenko I, Blanpain C: EMT Transition States during Tumor Progression and Metastasis. TRENDS CELL BIOL 2019, 29(3):212-226.

22. Serrano-Gomez SJ, Maziveyi M, Alahari SK: Regulation of epithelial-mesenchymal transition through epigenetic and post-translational modifications. MOL CANCER 2016, 15:18.

23. Thierauf $\mathrm{J}$, Veit $\mathrm{J}$, Hess $\mathrm{J}$ : Epithelial-to-Mesenchymal Transition in the Pathogenesis and Therapy of Head and Neck Cancer. CANCERS 2017, 9(12):76.

24. Lamouille S, Xu J, Derynck R: Molecular mechanisms of epithelial-mesenchymal transition. NAT REV MOL CELL BIO 2014, 15(3):178-196.

25. Gruber J, Yee Z, Tolwinski N: Developmental Drift and the Role of Wnt Signaling in Aging. CANCERS 2016, 8(8):73.

26. Shi J, Chi S, Xue J, Yang J, Li F, Liu X: Emerging Role and Therapeutic Implication of Wnt Signaling Pathways in Autoimmune Diseases. J IMMUNOL RES 2016, 2016:1-18.

\section{Tables}

TABLE 1: Correlation between FAM83A expression and multiple clinicopathological parameters in HNSCC 


\begin{tabular}{|c|c|c|c|c|}
\hline \multirow[t]{2}{*}{ Pathologic characteristics } & \multirow[t]{2}{*}{$\mathrm{n}$} & \multicolumn{2}{|c|}{ FAM83A expression (number of cases) } & \multirow[t]{2}{*}{$P$ value } \\
\hline & & Low & High & \\
\hline \multicolumn{5}{|l|}{ Age, years } \\
\hline$\geq 60$ & 118 & 88 & 30 & 0.2412 \\
\hline$<60$ & 124 & 84 & 40 & \\
\hline \multicolumn{5}{|l|}{ Sex } \\
\hline Male & 148 & 115 & 33 & 0.0056 \\
\hline Female & 94 & 57 & 37 & \\
\hline \multicolumn{5}{|l|}{ Tumor size } \\
\hline T1 & 109 & 87 & 22 & 0.0399 \\
\hline $\mathrm{T} 2$ & 95 & 63 & 32 & \\
\hline T3 & 24 & 14 & 10 & \\
\hline $\mathrm{T} 4$ & 14 & 8 & 6 & \\
\hline \multicolumn{5}{|l|}{ Lymph node status } \\
\hline NO & 130 & 108 & 22 & $<0.0001$ \\
\hline N1 & 41 & 31 & 10 & \\
\hline N2 & 27 & 17 & 10 & \\
\hline N3 & 44 & 16 & 28 & \\
\hline \multicolumn{5}{|l|}{ Pathological grade } \\
\hline I & 136 & 99 & 37 & 0.2984 \\
\hline II & 81 & 53 & 28 & \\
\hline III & 25 & 20 & 5 & \\
\hline \multicolumn{5}{|l|}{ Clinical stage } \\
\hline I & 64 & 52 & 12 & 0.0983 \\
\hline II & 51 & 36 & 15 & \\
\hline III & 35 & 26 & 9 & \\
\hline IV & 92 & 58 & 34 & \\
\hline
\end{tabular}

Figures 
A

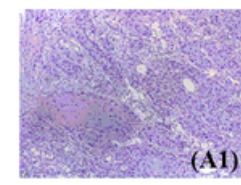

B

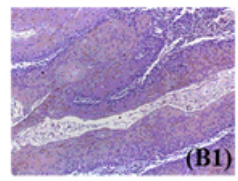

C

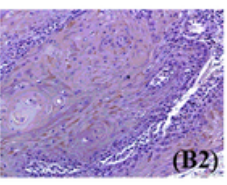

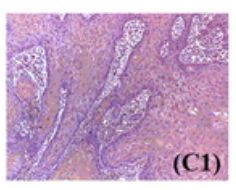

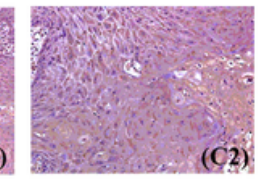

D

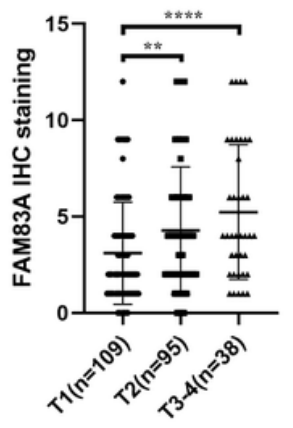

H

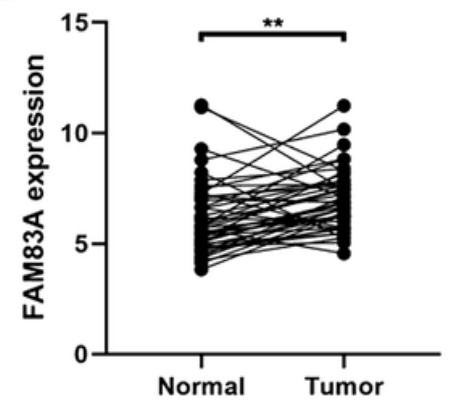

E

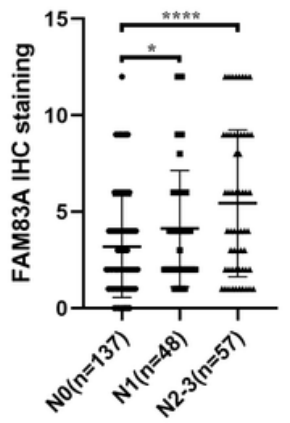

F

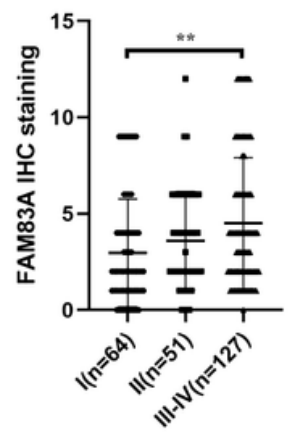

G

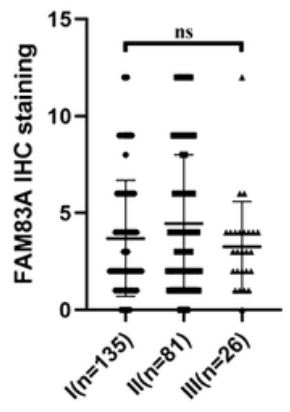

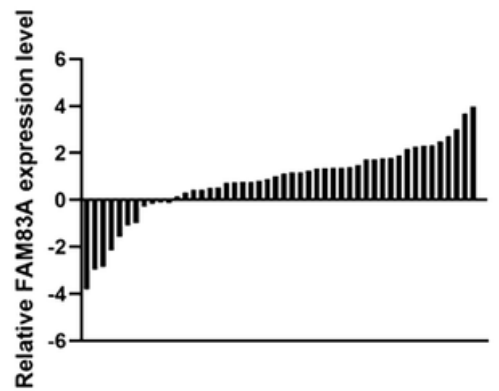

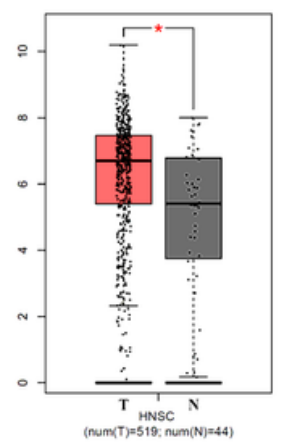

J

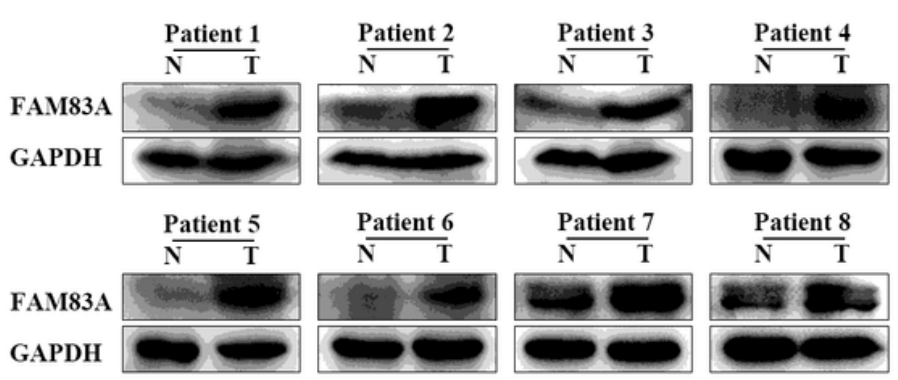

K

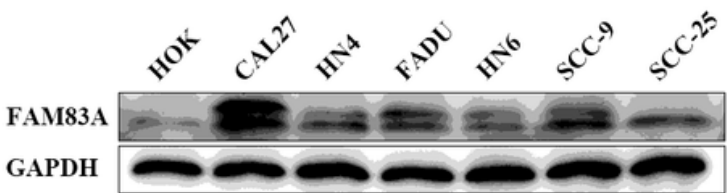

\section{Figure 1}

FAM83A is overexpressed and related to tumor size, lymph node metastasis, and pathological stage in HNSCC A. Negative FAM83A expression in HNSCC adjacent normal tissues (A1, 100x; A2, 200x). B. Low FAM83A expression in HNSCC tumor tissues (B1, 100x; B2, 200x). C. High FAM83A expression in HNSCC tumor tissues $(\mathrm{C} 1,100 \times ; \mathrm{C} 2,200 \times)$. D. Quantification analysis of FAM83A staining in HNSCC with different tumor size in microarrays. E. Quantification analysis of FAM83A staining in HNSCC with different lymph node metastasis in microarrays. F. Quantification analysis of FAM83A staining in HNSCC 
with different pathological stage in microarrays. G. Quantification analysis of FAM83A staining in HNSCC with different clinical tumor stage in microarrays. $\mathrm{H}$. The relative expression of FAM83A mRNA was measured by qRT-PCR in freshly collected HNSCC samples and paired adjacent non-tumor tissues. The height of column represents the fold change (log2-transformed) in FAM83A expression in these 48 patients (right panel). I. Differential expression of FAM83A in HNSCC $(n=519)$ and normal tissues ( $n$ $=44)$ from the TCGA database. J. FAM83A protein expression in eight HNSCC tissue and matched adjacent normal tissues. K. FAM83A protein expression in HOK and HNSCC cell lines. Data were statistically analyzed with Student's t-test and values are shown as the mean $\pm S D$. ${ }^{\star} p<0.05,{ }^{* \star} p<0.01$, $\star \star \star p<0.001$.

A

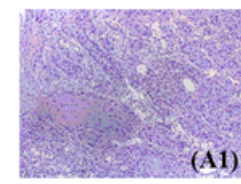

(A1)

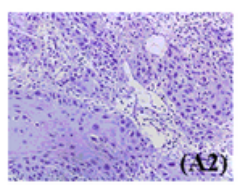

B

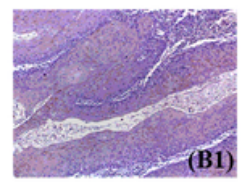

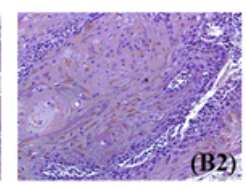

C
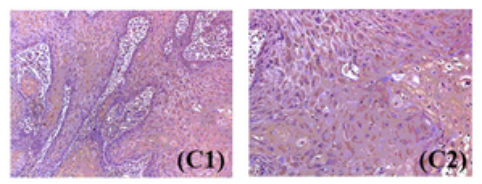

D

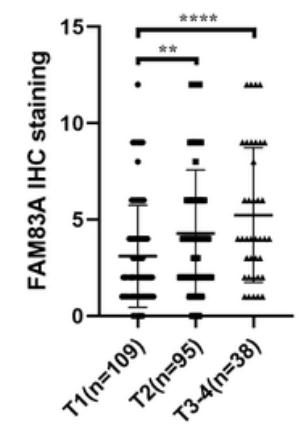

H

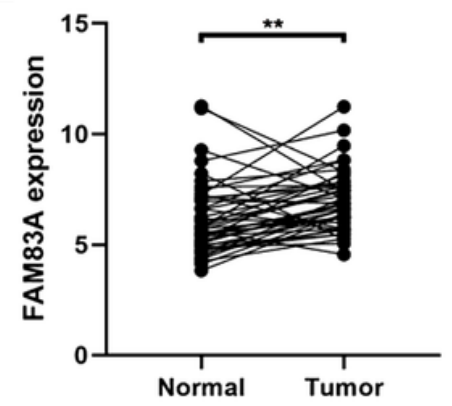

E

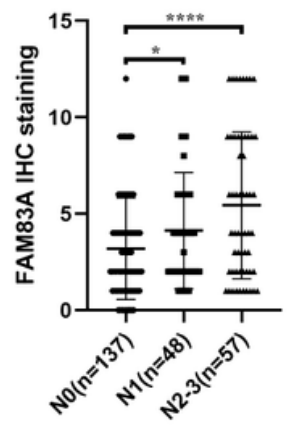

F

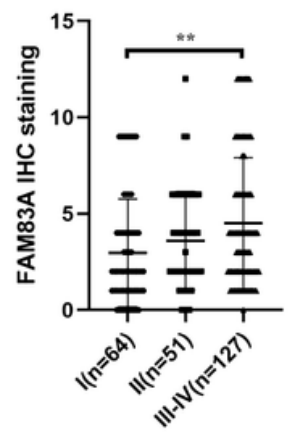

G

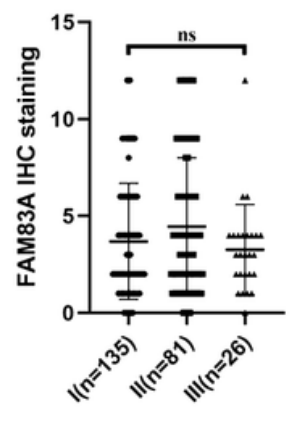

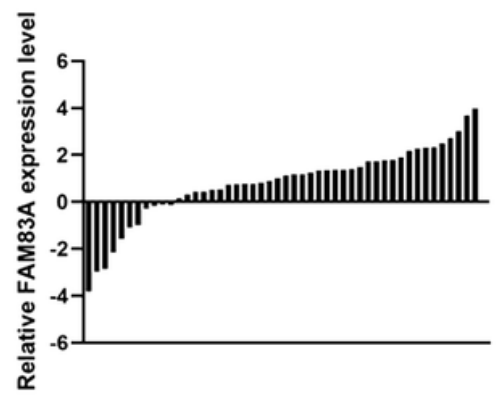

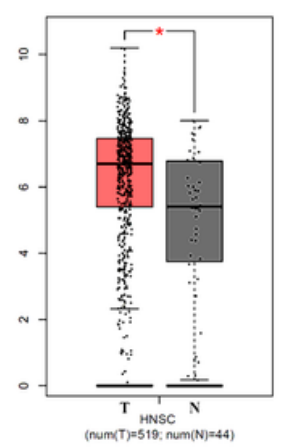

J

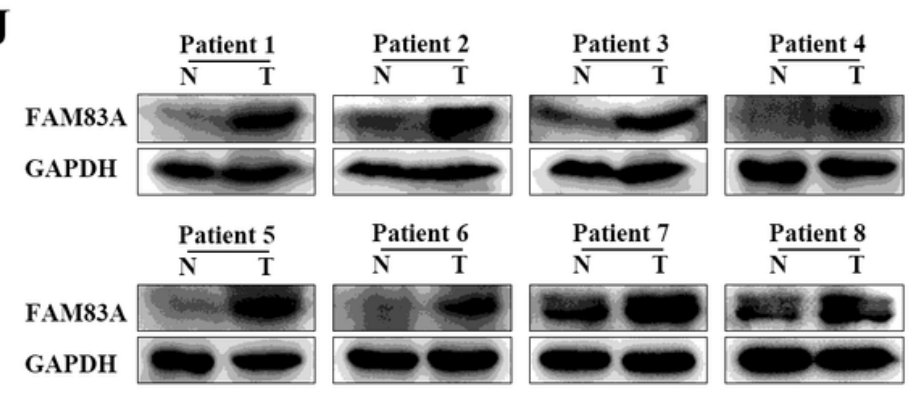

K

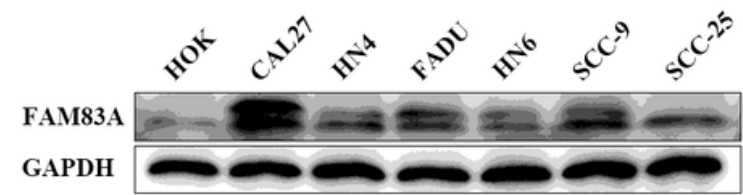




\section{Figure 1}

FAM83A is overexpressed and related to tumor size, lymph node metastasis, and pathological stage in HNSCC A. Negative FAM83A expression in HNSCC adjacent normal tissues (A1, 100x; A2, 200x). B. Low FAM83A expression in HNSCC tumor tissues (B1, 100x; B2, 200x). C. High FAM83A expression in HNSCC tumor tissues (C1, 100x; C2, 200x). D. Quantification analysis of FAM83A staining in HNSCC with different tumor size in microarrays. E. Quantification analysis of FAM83A staining in HNSCC with different lymph node metastasis in microarrays. F. Quantification analysis of FAM83A staining in HNSCC with different pathological stage in microarrays. G. Quantification analysis of FAM83A staining in HNSCC with different clinical tumor stage in microarrays. $\mathrm{H}$. The relative expression of FAM83A mRNA was measured by qRT-PCR in freshly collected HNSCC samples and paired adjacent non-tumor tissues. The height of column represents the fold change (log2-transformed) in FAM83A expression in these 48 patients (right panel). I. Differential expression of FAM83A in HNSCC $(n=519)$ and normal tissues ( $n$ $=44$ ) from the TCGA database. J. FAM83A protein expression in eight HNSCC tissue and matched adjacent normal tissues. K. FAM83A protein expression in HOK and HNSCC cell lines. Data were statistically analyzed with Student's t-test and values are shown as the mean $\pm S D$. ${ }^{\star} p<0.05,{ }^{\star \star} p<0.01$, $\star \star \star p<0.001$. 

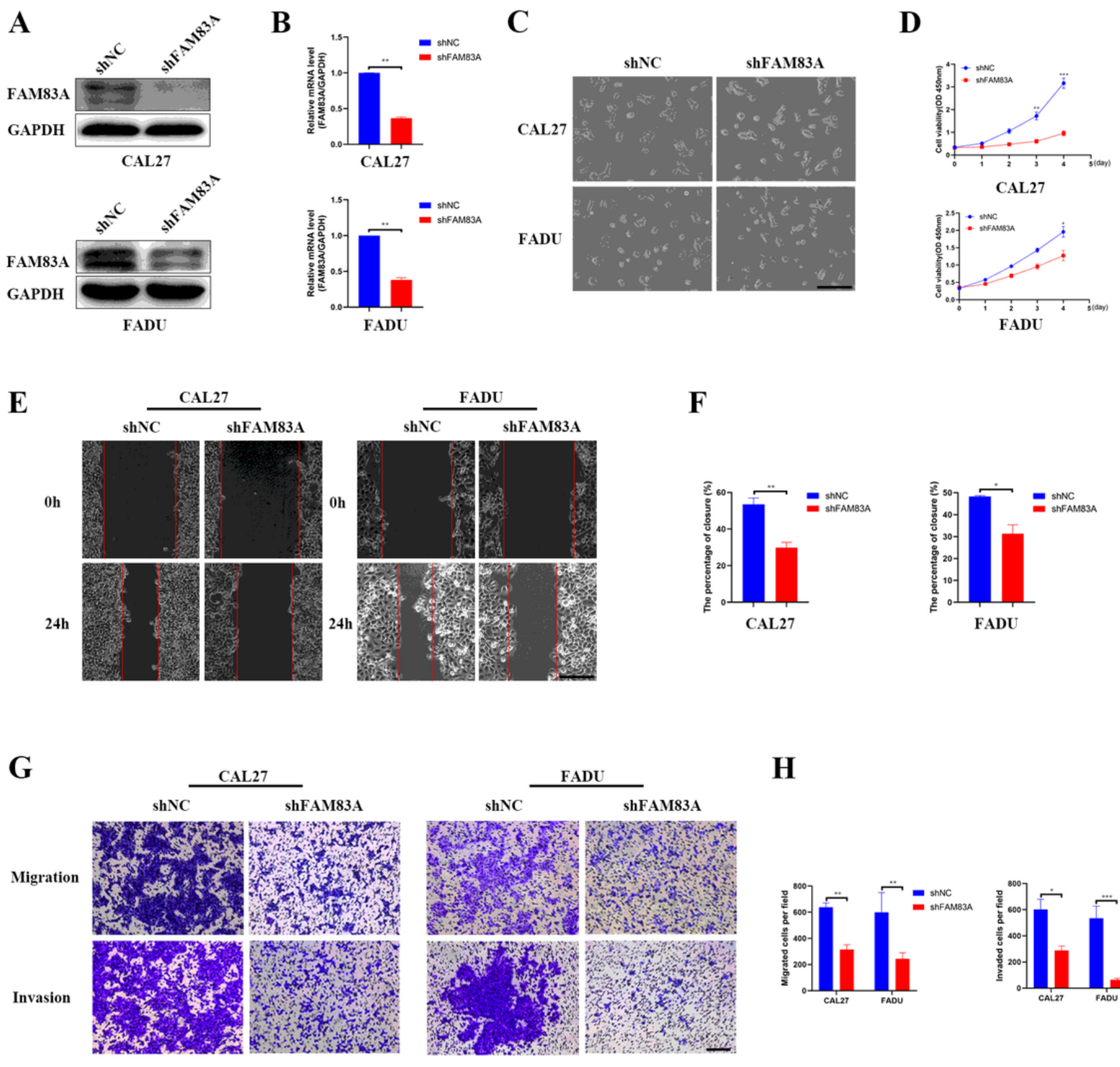

$\mathbf{H}$
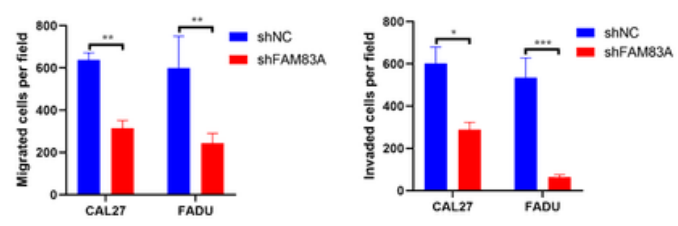

\section{Figure 2}

FAM83A knockdown in CAL27 and FADU cells inhibited cell growth and metastasis. A. B. FAM83A expression based on real-time PCR and western blotting in CAL27 and FADU cell lines after shFAM83A transfection. C. Morphological changes of Cal27 and FADU cells after shFAM83A transfection. D. CCK8 assays of the proliferation in CAL27 and FADU cells after shFAM83A transfection. E.F. A wound-healing assay in CAL27 and FADU cells after shFAM83A transfection. G.H. Transwell assays of migration and 
invasion in CAL27 and FADU cells after shFAM83A transfection. Data were statistically analyzed with Student's t-test and values are shown as the mean \pm SD. ${ }^{*} p<0.05,{ }^{* *} p<0.01,{ }^{* * *} p<0.001$.

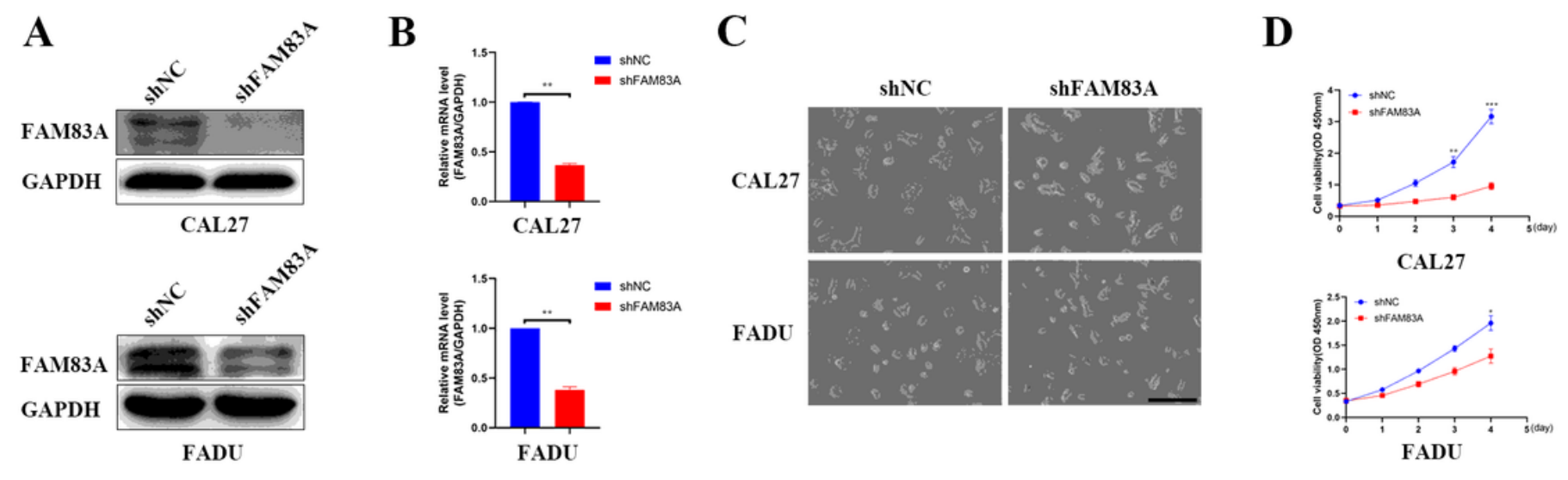

$\mathbf{E}$
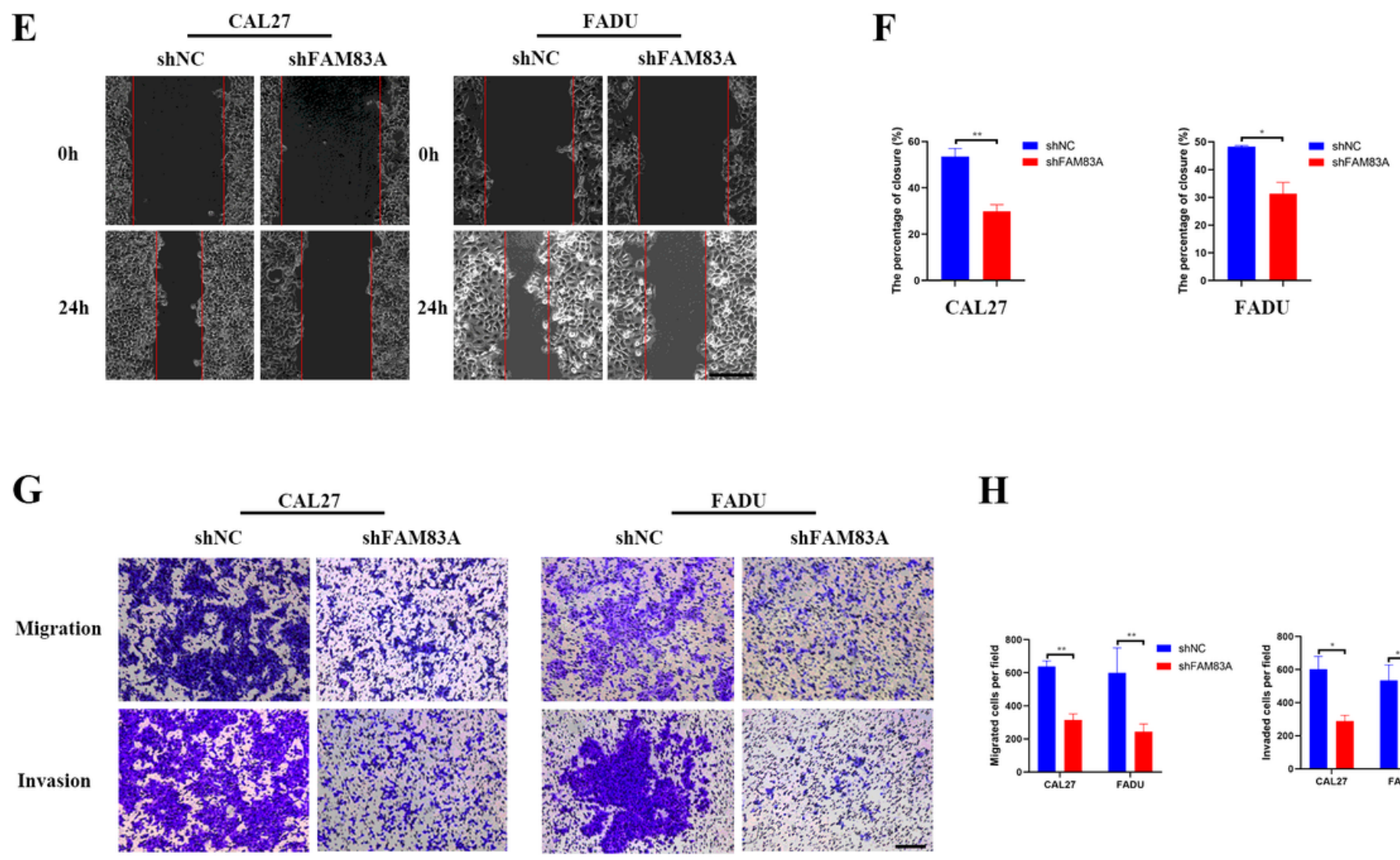

$\mathbf{H}$
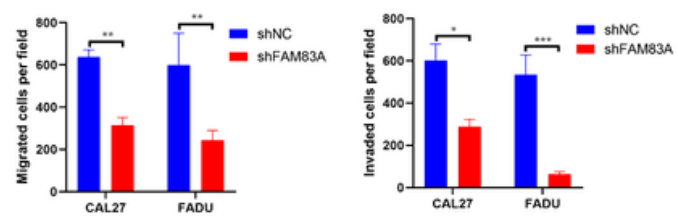

Figure 2

FAM83A knockdown in CAL27 and FADU cells inhibited cell growth and metastasis. A. B. FAM83A expression based on real-time PCR and western blotting in CAL27 and FADU cell lines after shFAM83A transfection. C. Morphological changes of Cal27 and FADU cells after shFAM83A transfection. D. CCK8 assays of the proliferation in CAL27 and FADU cells after shFAM83A transfection. E.F. A wound-healing 
assay in CAL27 and FADU cells after shFAM83A transfection. G.H. Transwell assays of migration and invasion in CAL27 and FADU cells after shFAM83A transfection. Data were statistically analyzed with Student's t-test and values are shown as the mean \pm SD. ${ }^{*} p<0.05,{ }^{* *} p<0.01,{ }^{* * *} p<0.001$.
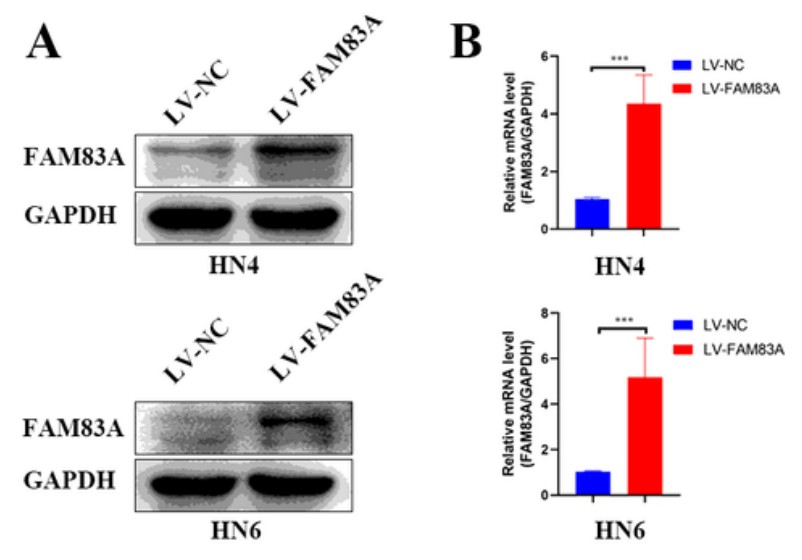

C

D
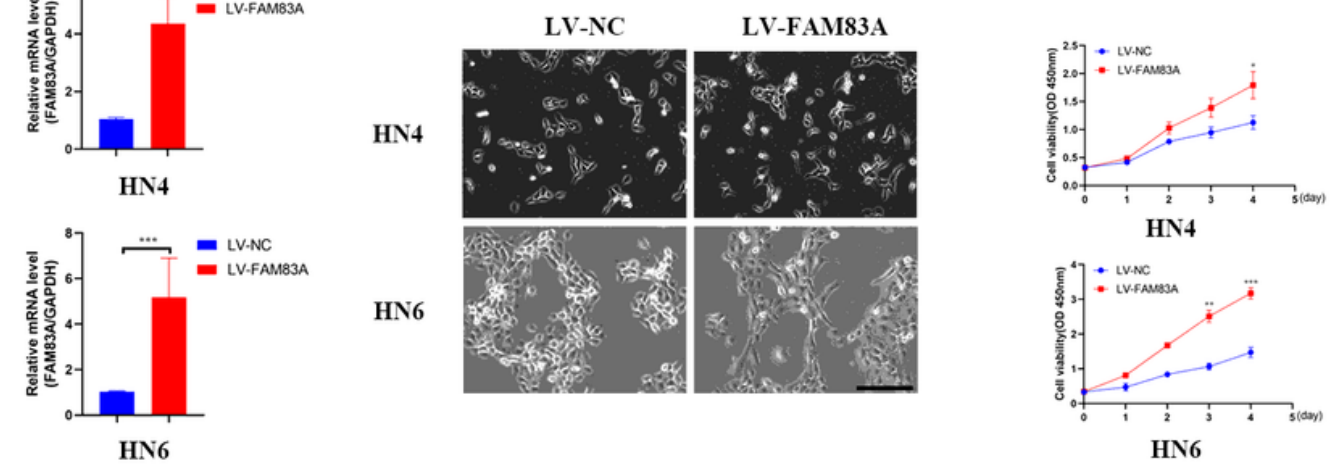

$\mathbf{E}$
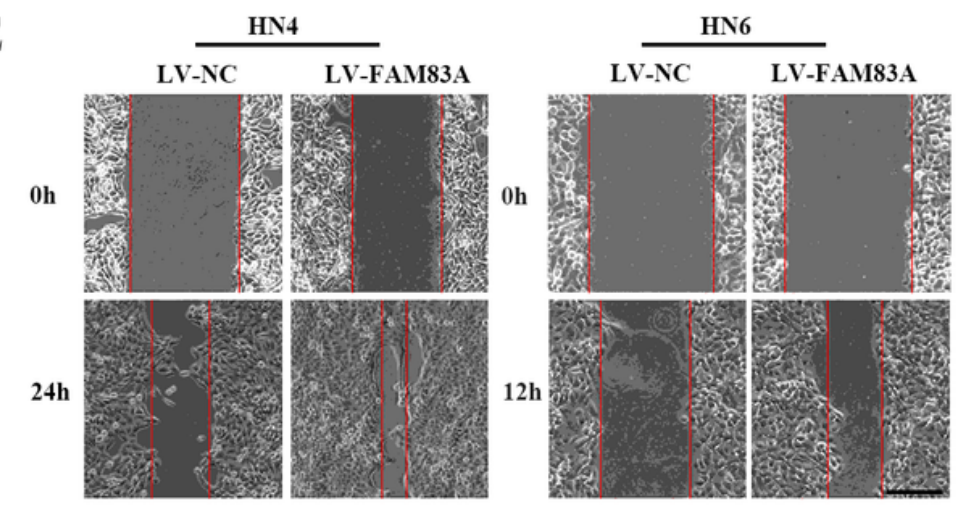

$\mathbf{F}$
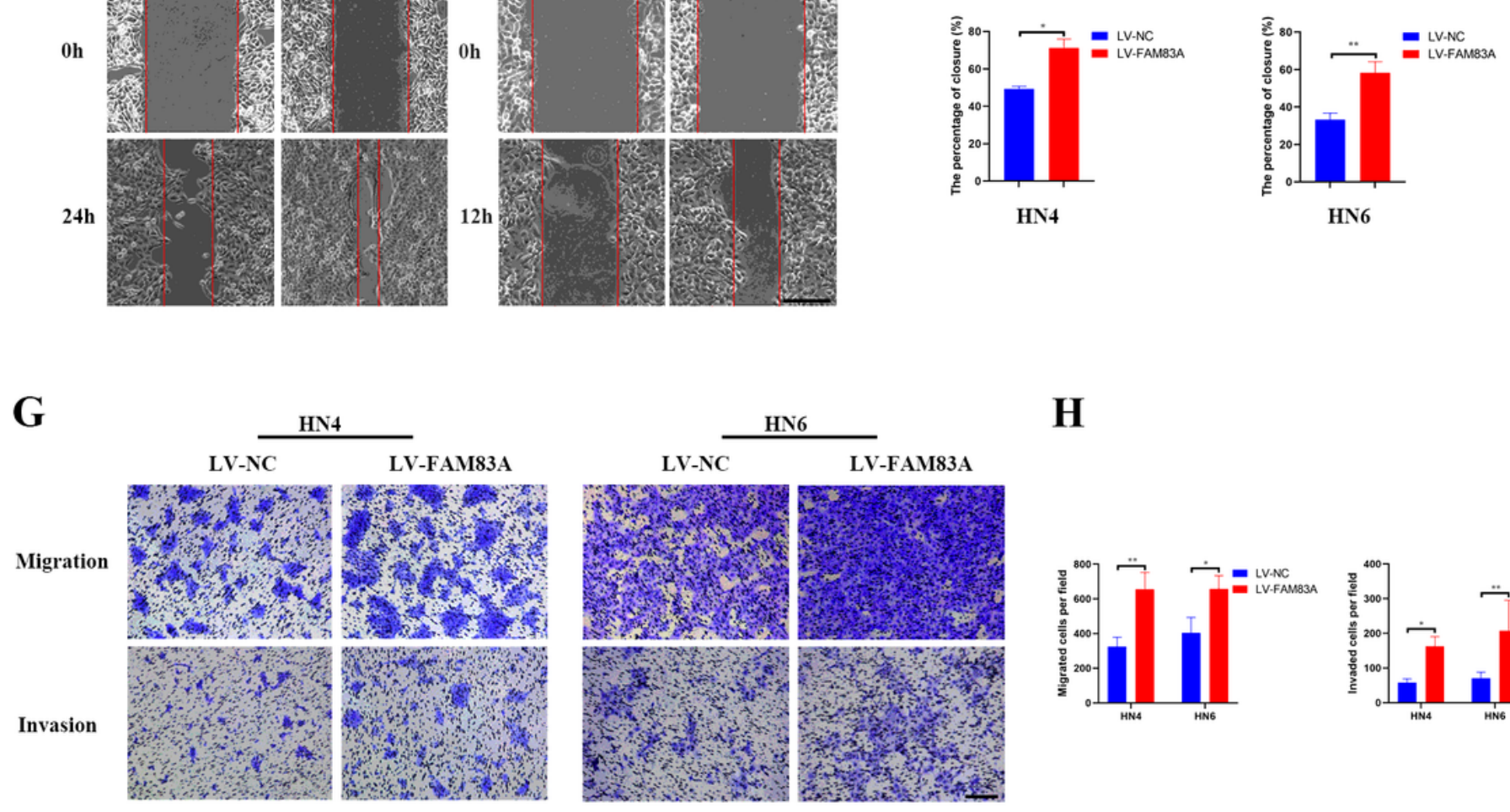

$\mathbf{H}$
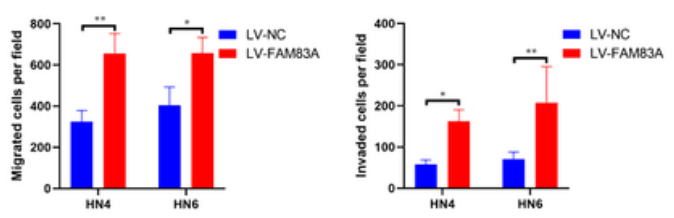

Figure 3

FAM83A overexpression in HN4 and HN6 cells promoted cell growth and metastasis. A. B. FAM83A expression based on real-time PCR and western blotting in HN4 and HN6 cell lines after treated with LVFAM83A. C. Morphological changes of HN4 and HN6 cells after LV-FAM83A transfection. D. CCK8 assays 
of the proliferation in HN4 and HN6 cells after LV-FAM83A transfection. E.F. A wound-healing assay in HN4 and HN6 cells after LV-FAM83A transfection. G.H. Transwell assays of migration and invasion in HN4 and HN6 cells after LV-FAM83A transfection. Data were statistically analyzed with Student's t-test and values are shown as the mean \pm SD. ${ }^{*} p<0.05,{ }^{* *} p<0.01,{ }^{* *} p<0.001$.
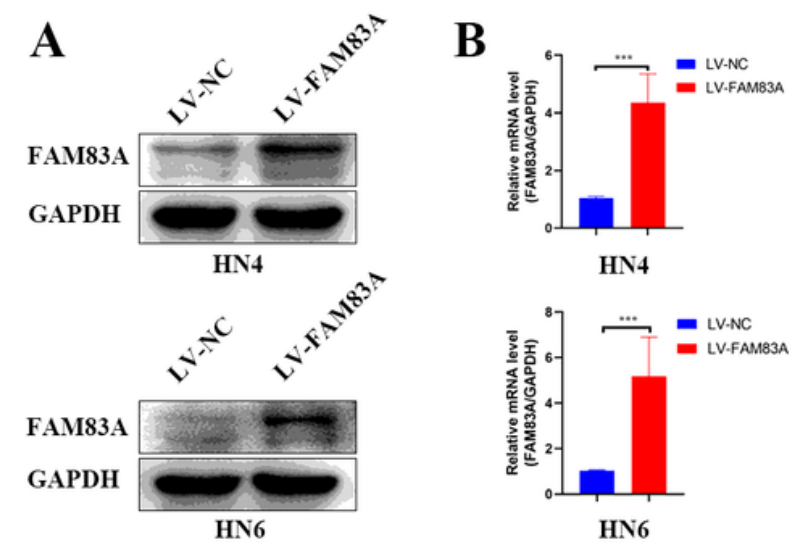

C

D
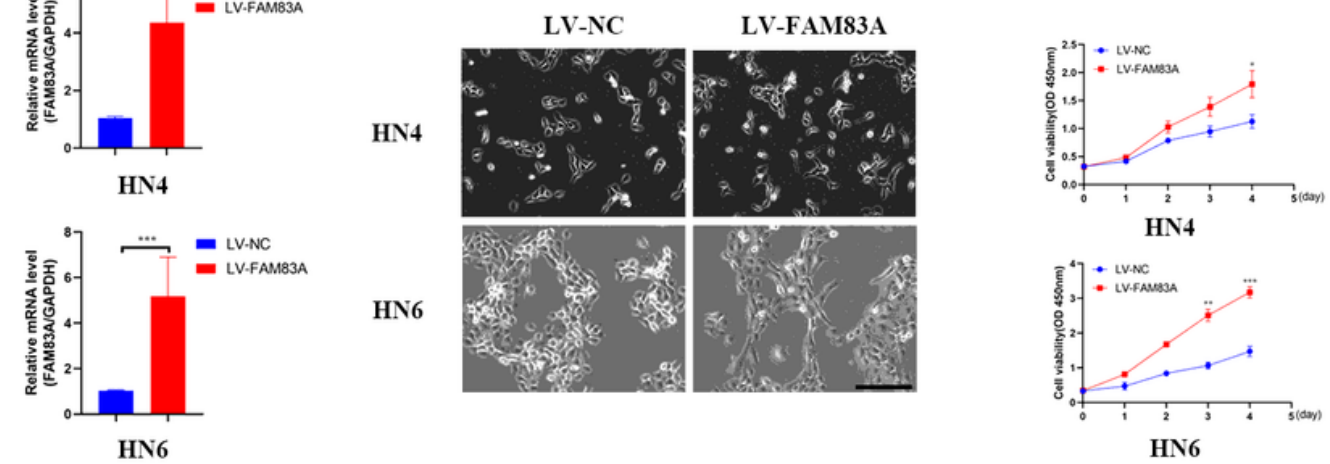

$\mathbf{E}$

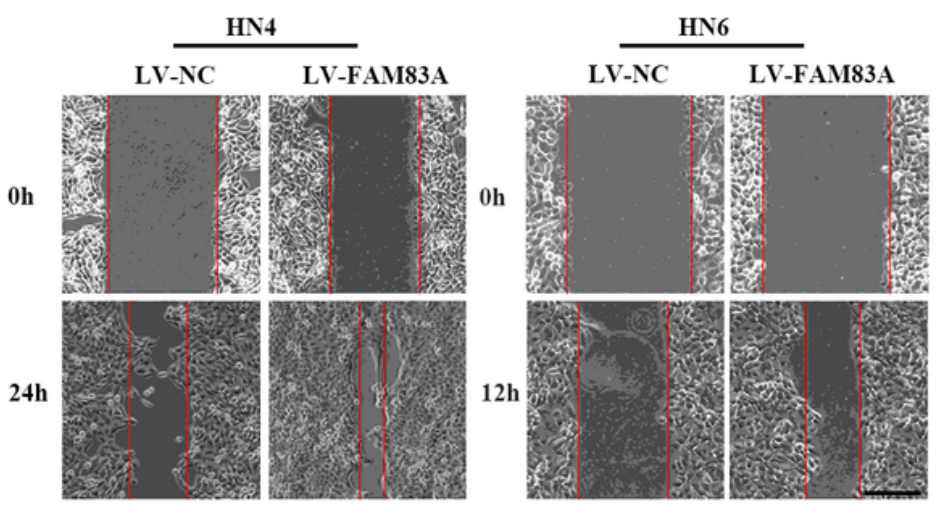

F
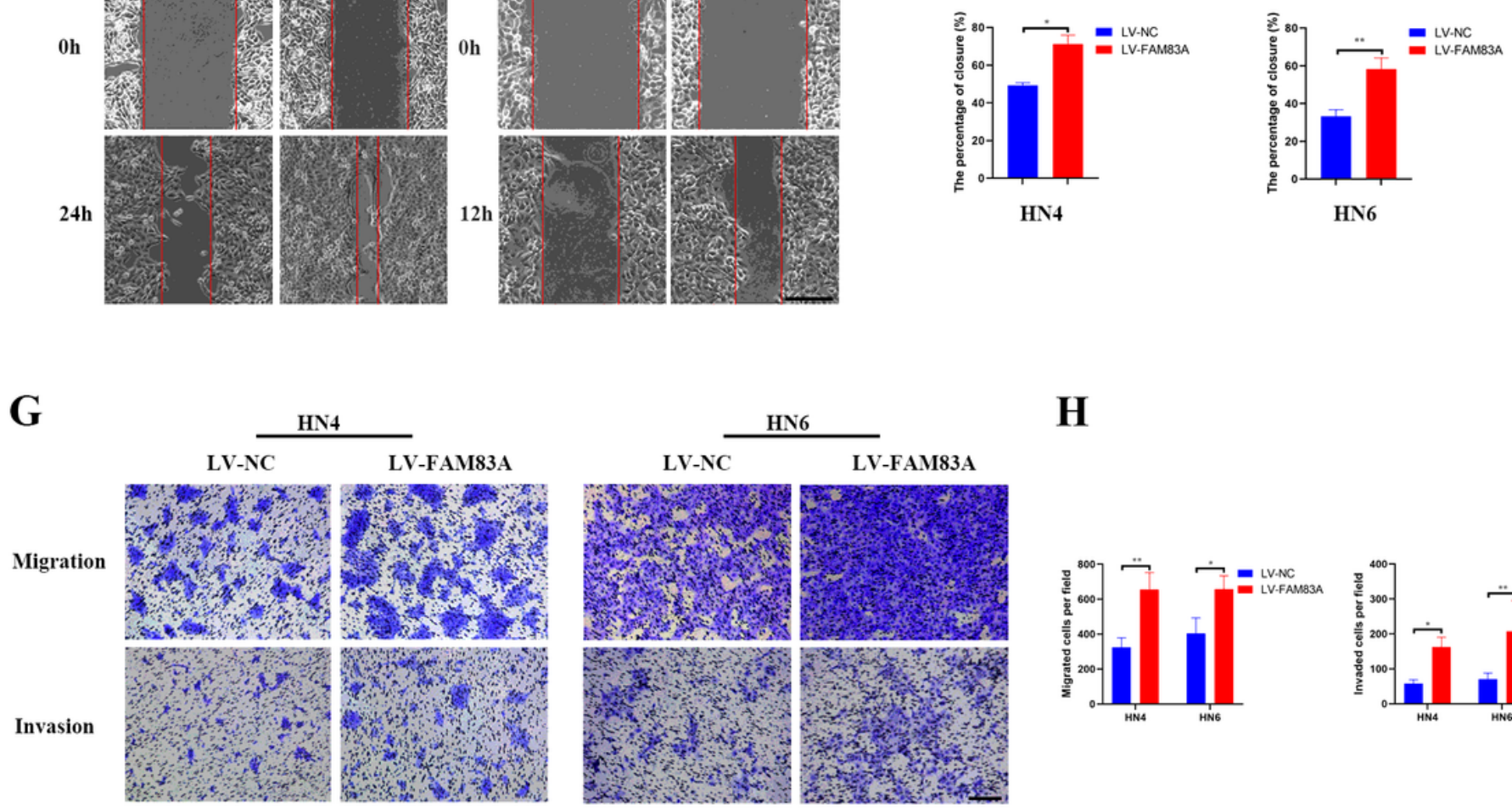

$\mathbf{H}$
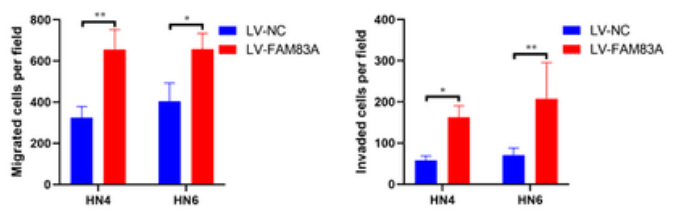

Figure 3

FAM83A overexpression in HN4 and HN6 cells promoted cell growth and metastasis. A. B. FAM83A expression based on real-time PCR and western blotting in HN4 and HN6 cell lines after treated with LV- 
FAM83A. C. Morphological changes of HN4 and HN6 cells after LV-FAM83A transfection. D. CCK8 assays of the proliferation in HN4 and HN6 cells after LV-FAM83A transfection. E.F. A wound-healing assay in HN4 and HN6 cells after LV-FAM83A transfection. G.H. Transwell assays of migration and invasion in HN4 and HN6 cells after LV-FAM83A transfection. Data were statistically analyzed with Student's t-test and values are shown as the mean \pm SD. ${ }^{\star} p<0.05,{ }^{\star \star} p<0.01,{ }^{\star \star \star} p<0.001$.
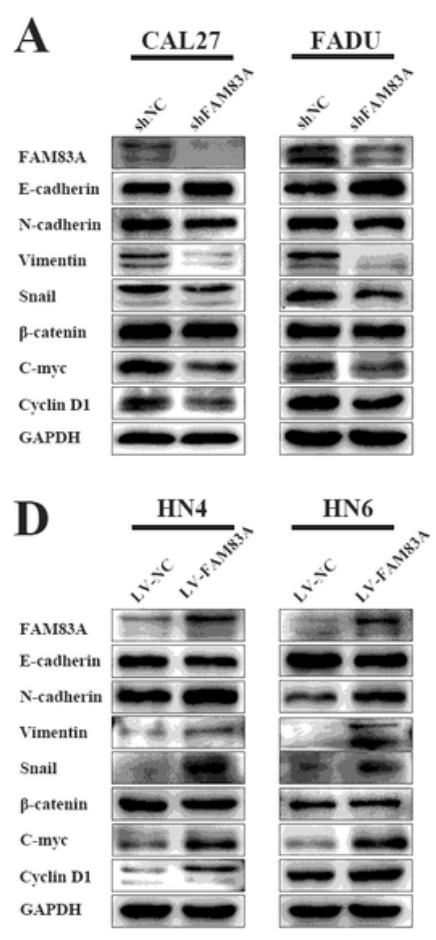

B

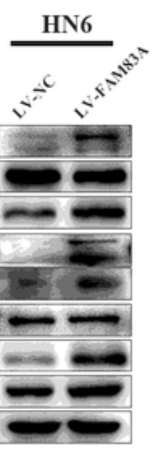

$\mathbf{E}$
CAL27

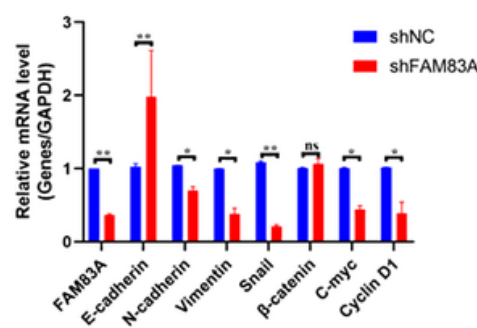

HN4

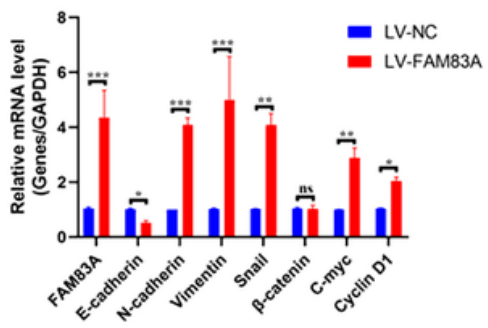

FADU

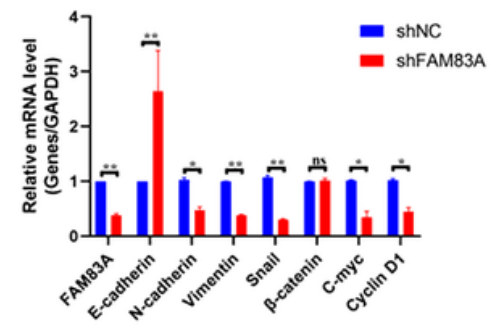

$\mathbf{F}$

HN6

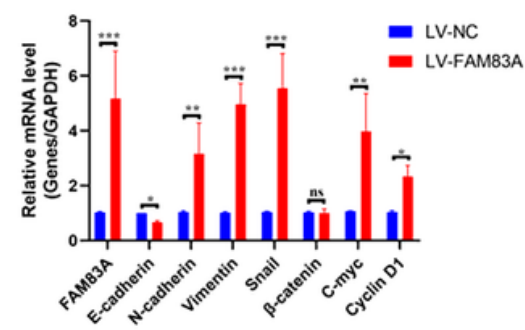

G

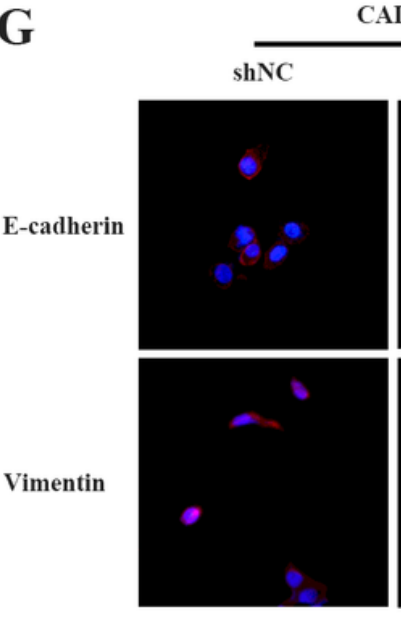

shFAM83A
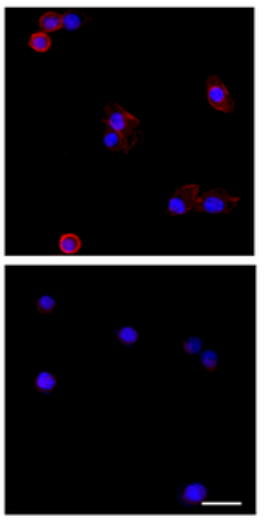

H

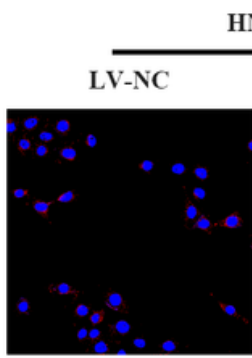

HN6

E-cadherin

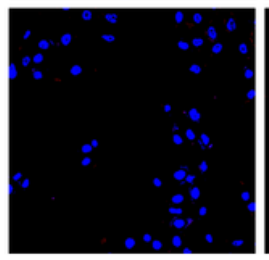

Vimentin

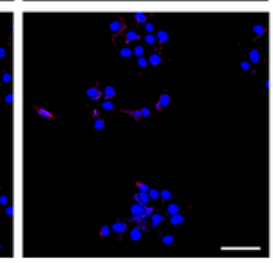

Figure 4 
FAM83A promoted EMT in HNSCC cell lines. A. Protein levels of FAM83A, E-cadherin, N-cadherin, Vimentin, Snail and Wnt/ $\beta$-catenin pathway downstream targets $\mathrm{C}$-myc and Cyclin D1 were determined by western blotting in CAL27 and FADU cells after shFAM83A transfection. B.C. Gene expression of FAM83A, E-cadherin, N-cadherin, Vimentin, Snail and Wnt/ $\beta$-catenin pathway downstream targets $\mathrm{C}$-myc and Cyclin D1 were measured by real-time RT-PCR in CAL27 and FAUD cells after shFAM83A transfection. D. Protein levels of FAM83A, E-cadherin, N-cadherin, Vimentin, Snail and Wnt/ $\beta$-catenin pathway downstream targets C-myc and Cyclin D1 were determined by western blotting in HN4 and HN6 cells after LV-FAM83A transfection. E.F. Gene expression of FAM83A, E-cadherin, N-cadherin, Vimentin, Snail and Wnt/ $\beta$-catenin pathway downstream targets C-myc and Cyclin D1 were measured by real-time RT-PCR in HN4 and HN6 cells after LV-FAM83A transfection. G. Immunofluorescence analysis of the EMT marker E-cadherin and Vimentin in CAL27 cells after shFAM83A transfection. H. Immunofluorescence analysis of the EMT marker E-cadherin and Vimentin in HN6 cells after LV-FAM83A transfection. Data were statistically analyzed with Student's t-test and values are shown as the mean \pm SD. ${ }^{\star} p<0.05,{ }^{\star \star} p<0.01,{ }^{\star \star *} p<0.001$. 


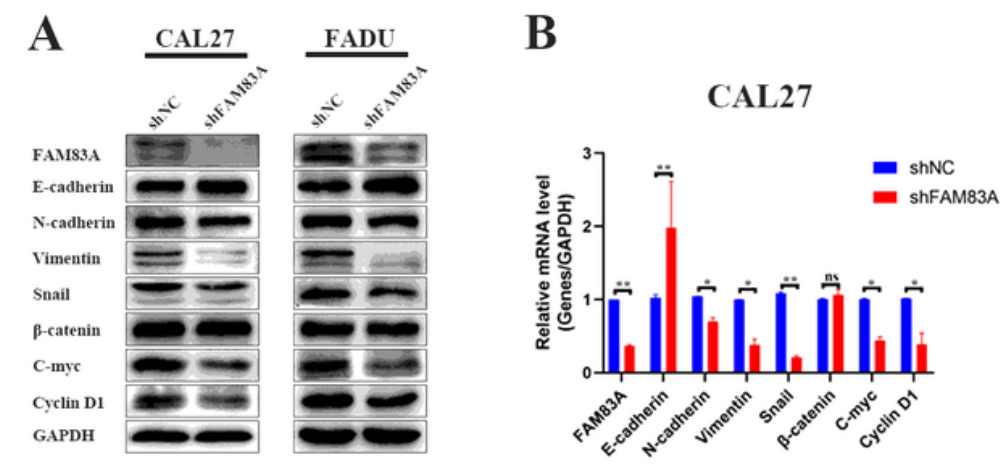

C
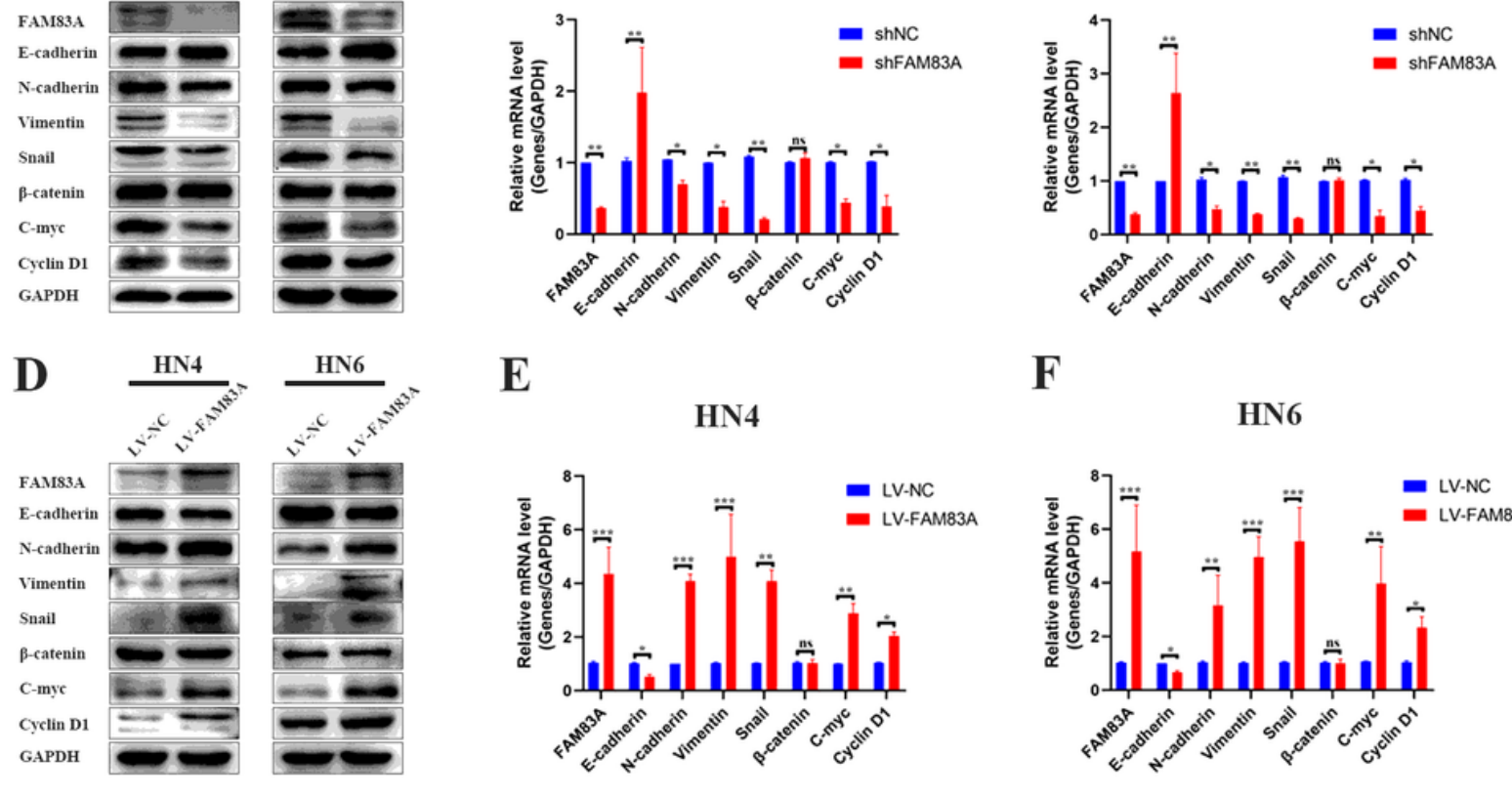

$\mathbf{F}$
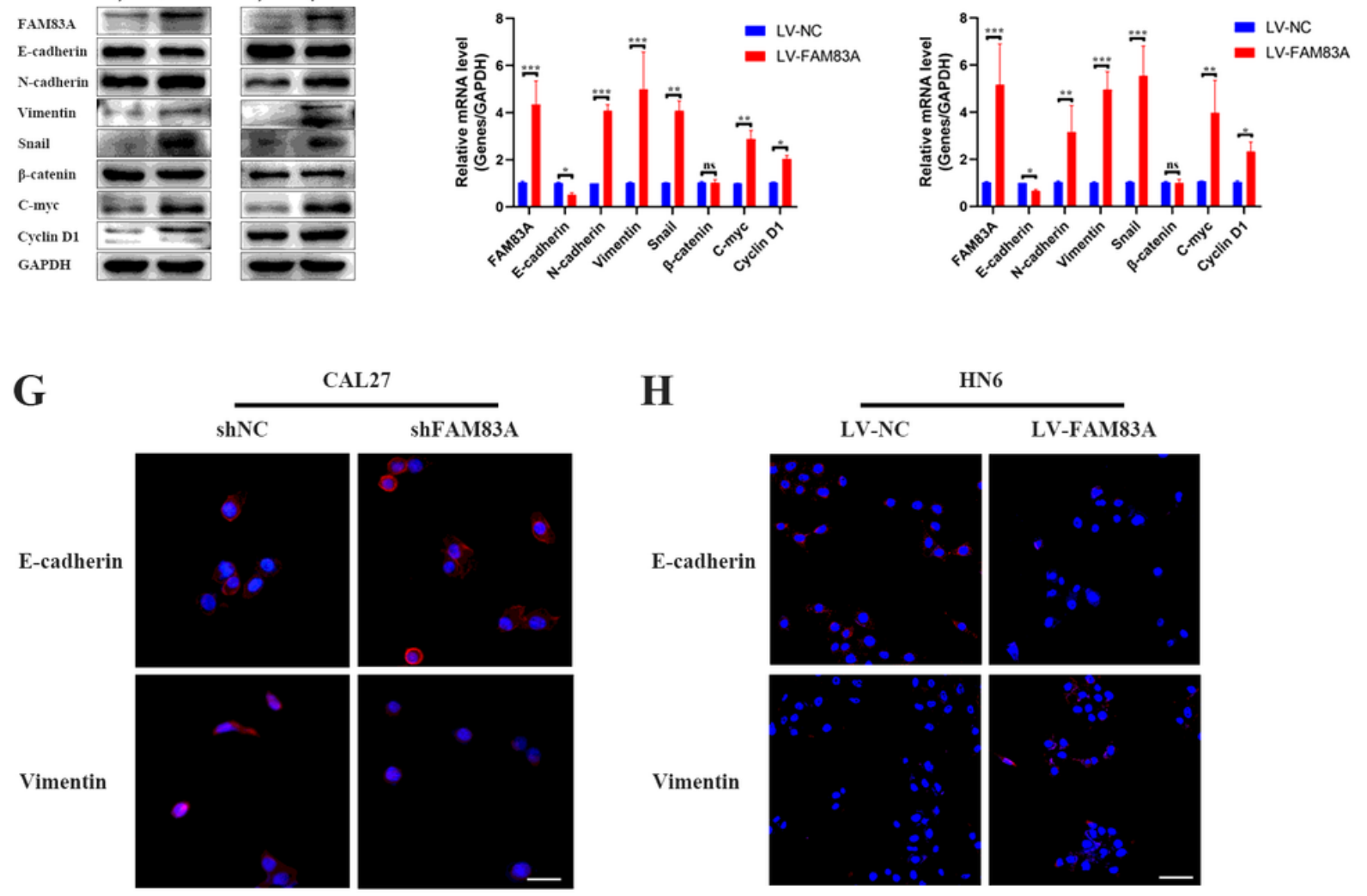

\section{Figure 4}

FAM83A promoted EMT in HNSCC cell lines. A. Protein levels of FAM83A, E-cadherin, N-cadherin, Vimentin, Snail and Wnt/ $\beta$-catenin pathway downstream targets C-myc and Cyclin D1 were determined by western blotting in CAL27 and FADU cells after shFAM83A transfection. B.C. Gene expression of FAM83A, E-cadherin, N-cadherin, Vimentin, Snail and Wnt/ $\beta$-catenin pathway downstream targets C-myc and Cyclin D1 were measured by real-time RT-PCR in CAL27 and FAUD cells after shFAM83A transfection. D. Protein levels of FAM83A, E-cadherin, N-cadherin, Vimentin, Snail and Wnt/ $\beta$-catenin pathway downstream 
targets C-myc and Cyclin D1 were determined by western blotting in HN4 and HN6 cells after LV-FAM83A transfection. E.F. Gene expression of FAM83A, E-cadherin, N-cadherin, Vimentin, Snail and Wnt/ $\beta$-catenin pathway downstream targets C-myc and Cyclin D1 were measured by real-time RT-PCR in HN4 and HN6 cells after LV-FAM83A transfection. G. Immunofluorescence analysis of the EMT marker E-cadherin and Vimentin in CAL27 cells after shFAM83A transfection. H. Immunofluorescence analysis of the EMT marker E-cadherin and Vimentin in HN6 cells after LV-FAM83A transfection. Data were statistically analyzed with Student's t-test and values are shown as the mean \pm SD. ${ }^{*} p<0.05,{ }^{*} p<0.01,{ }^{* *} p<0.001$.
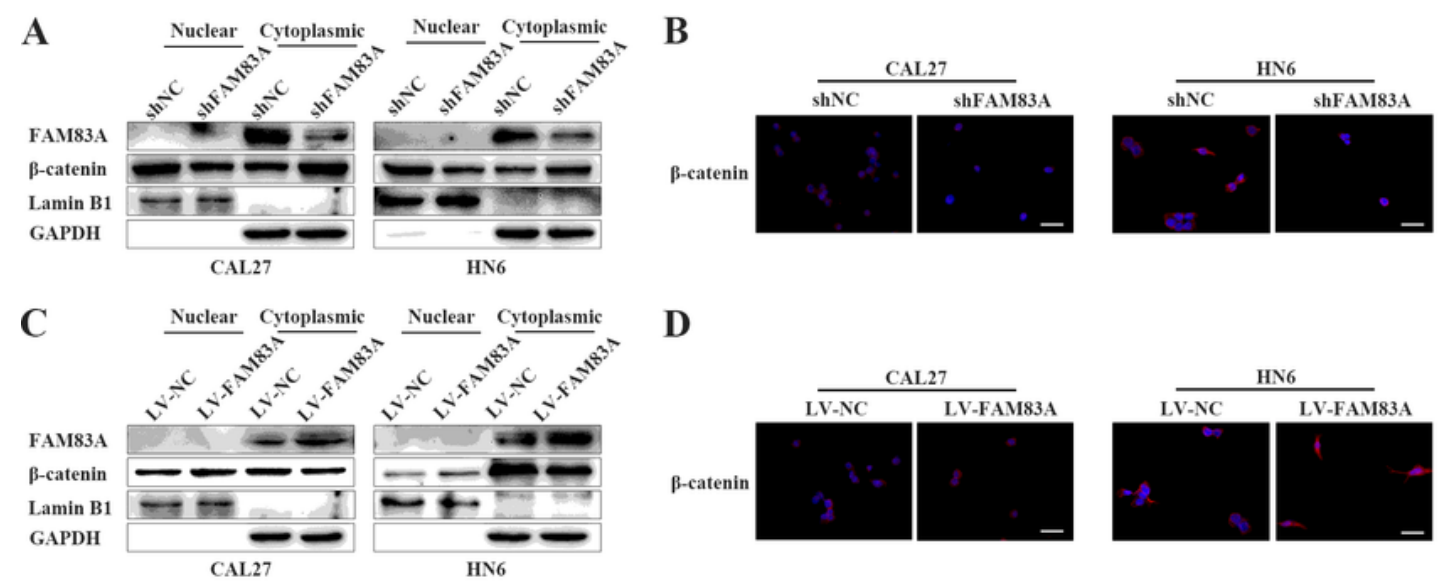

D

E
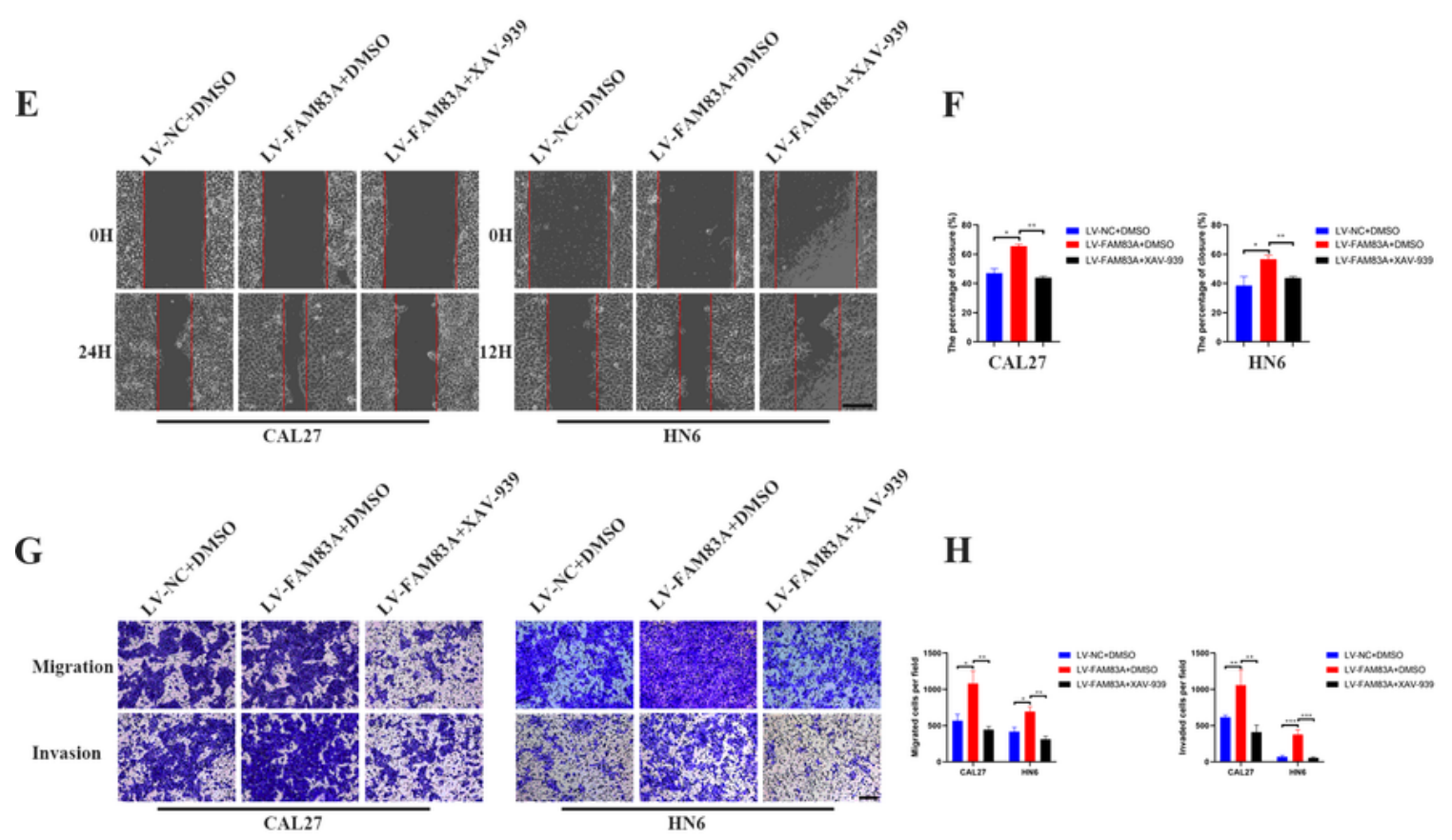

I
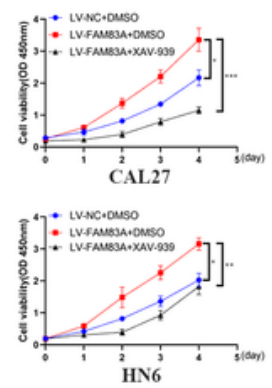

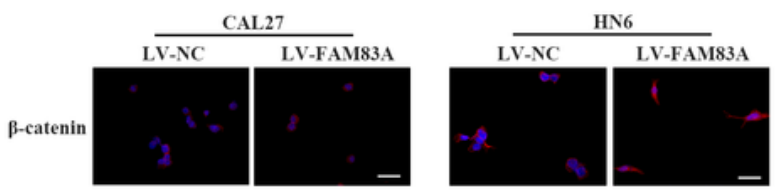

$\mathbf{H}$

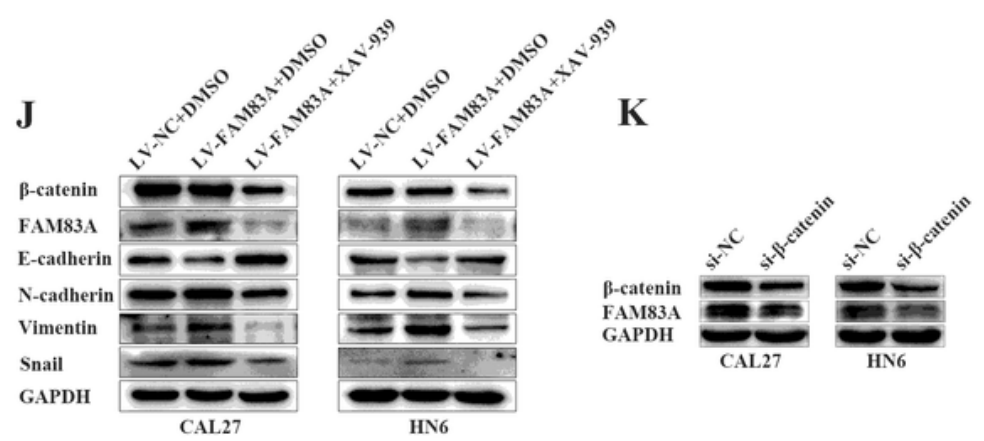




\section{Figure 5}

FAM83A enhanced Wnt/ $\beta$-catenin pathway activation in HNSCC and $\beta$-catenin downregulation affected FAM83A expression. A. Expression of nuclear and cytoplasmic FAM83A and $\beta$-catenin protein in CAL27 and HN6 cells after FAM83A silencing. B. Immunofluorescence assay of $\beta$-catenin in CAL27 and HN6 cells after FAM83A silencing. $C$. Expression of nuclear and cytoplasmic FAM83A and $\beta$-catenin protein in CAL27 and HN6 cells after FAM83A overexpressed. D. Immunofluorescence assay of $\beta$-catenin in CAL27 and HN6 cells after FAM83A overexpressed. E.F. A wound-healing assay in CAL27 and HN6 cells after treated with XAV-939. G.H. Transwell assays of migration and invasion in CAL27 and HN6 cells after treated with XAV-939 I. CCK8 assays of the proliferation in CAL27 and HN6 cells after treated with XAV939. J. Protein levels of $\beta$-catenin, FAM83A, E-cadherin, N-cadherin, Vimentin, and Snail and were determined by western blotting in CAL27 and HN6 cells after treated with XAV-939. K. Protein levels of $\beta$ catenin and FAM83A were determined by western blotting in CAL27 and HN6 cells after si- $\beta$-catenin transfection. Data were statistically analyzed with Student's t-test and values are shown as the mean \pm SD. ${ }^{*} p<0.05,{ }^{* *} p<0.01,{ }^{* *} p<0.001$. 

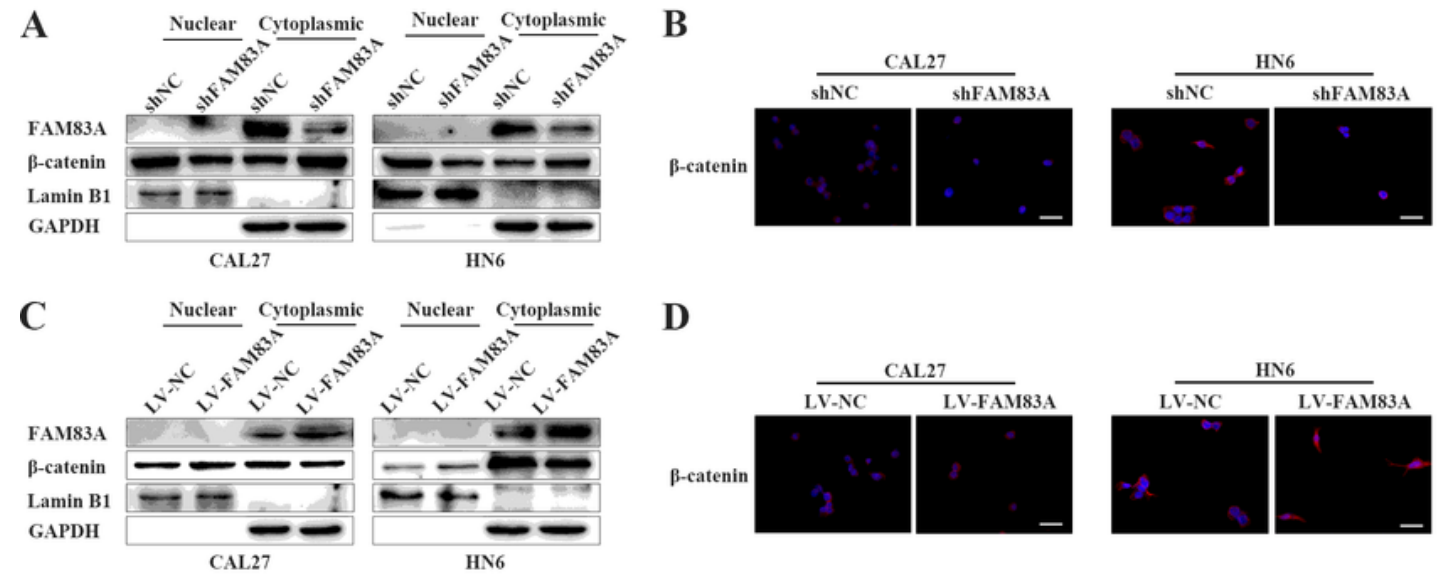

D

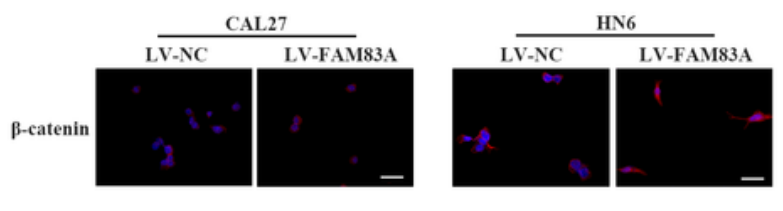

$\mathbf{E}$
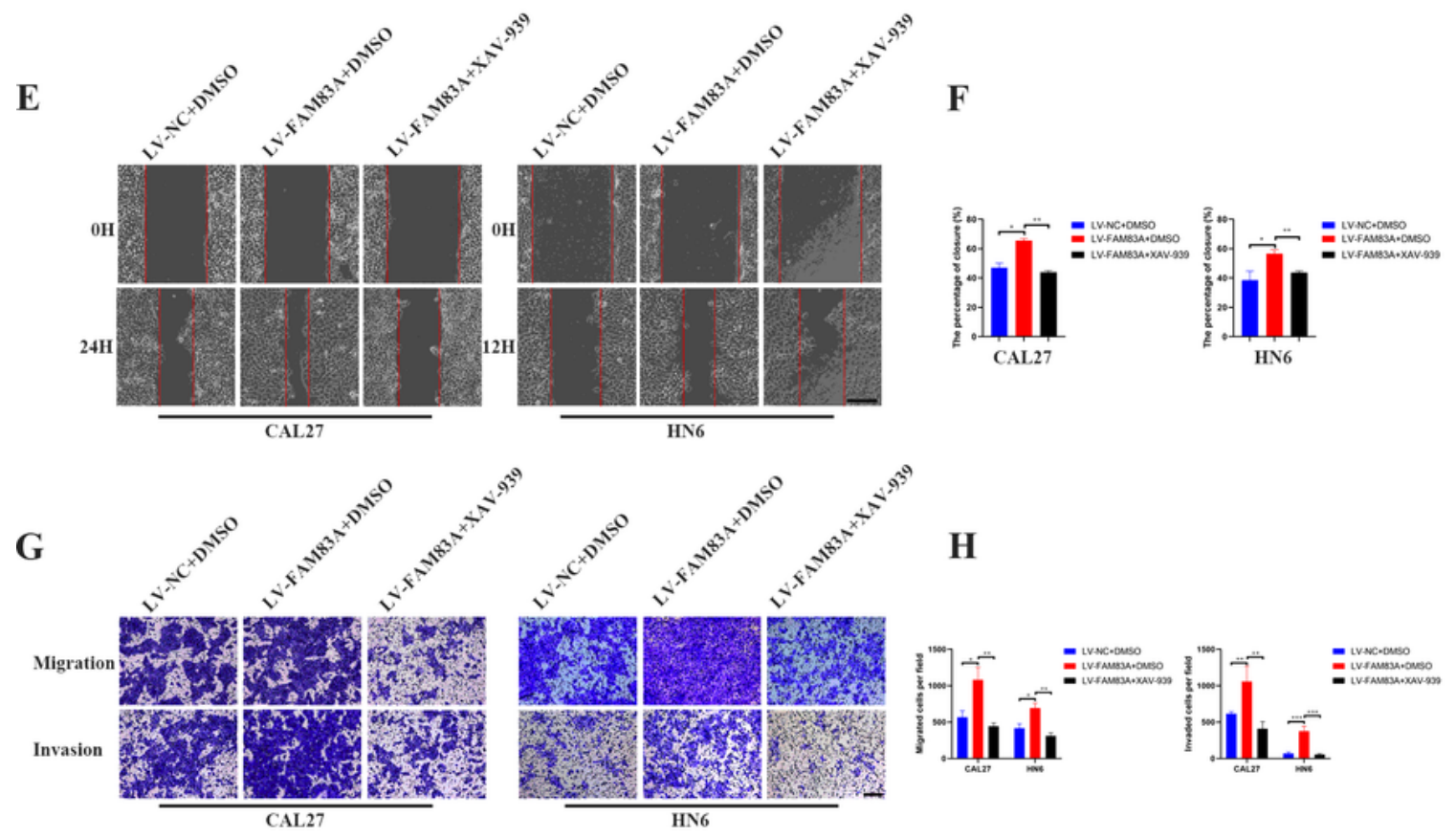

I

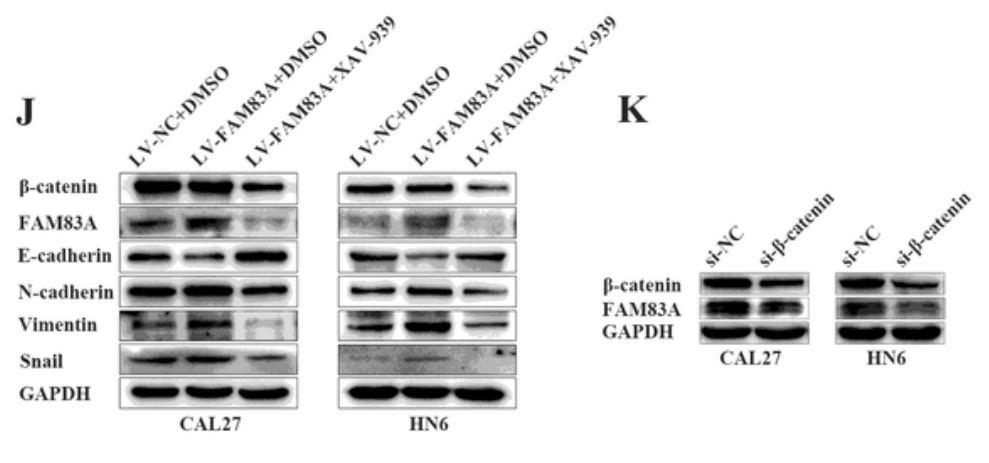

\section{Figure 5}

FAM83A enhanced Wnt/ $\beta$-catenin pathway activation in HNSCC and $\beta$-catenin downregulation affected FAM83A expression. A. Expression of nuclear and cytoplasmic FAM83A and $\beta$-catenin protein in CAL27 and HN6 cells after FAM83A silencing. B. Immunofluorescence assay of $\beta$-catenin in CAL27 and HN6 cells after FAM83A silencing. C. Expression of nuclear and cytoplasmic FAM83A and $\beta$-catenin protein in CAL27 and HN6 cells after FAM83A overexpressed. D. Immunofluorescence assay of $\beta$-catenin in CAL27 
and HN6 cells after FAM83A overexpressed. E.F. A wound-healing assay in CAL27 and HN6 cells after treated with XAV-939. G.H. Transwell assays of migration and invasion in CAL27 and HN6 cells after treated with XAV-939 I. CCK8 assays of the proliferation in CAL27 and HN6 cells after treated with XAV939. J. Protein levels of $\beta$-catenin, FAM83A, E-cadherin, N-cadherin, Vimentin, and Snail and were determined by western blotting in CAL27 and HN6 cells after treated with XAV-939. K. Protein levels of $\beta$ catenin and FAM83A were determined by western blotting in CAL27 and HN6 cells after si- $\beta$-catenin transfection. Data were statistically analyzed with Student's t-test and values are shown as the mean \pm SD. ${ }^{\star} p<0.05,{ }^{* \star} p<0.01,{ }^{\star \star *} p<0.001$.

A

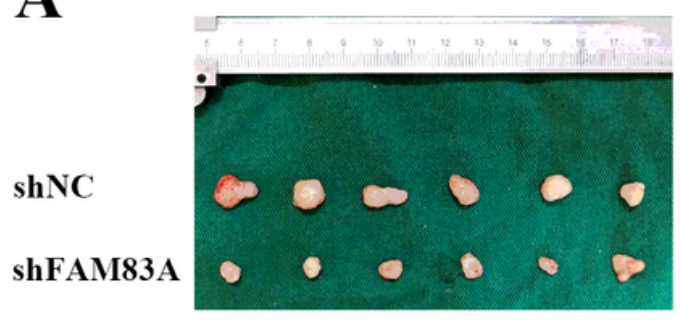

B

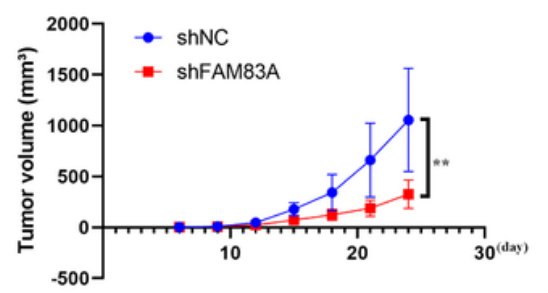

C

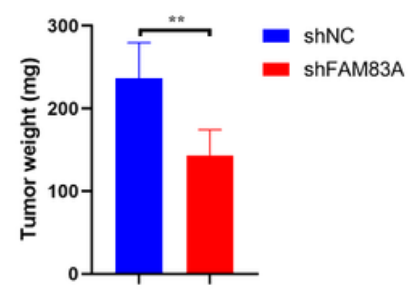

D

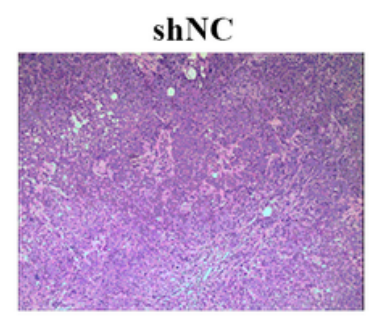

F

shNC

ShFAM83A
G

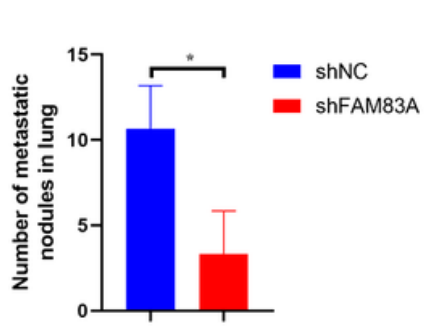

E

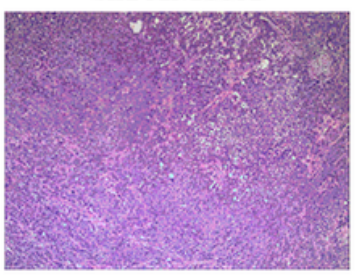

shNC

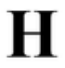

shFAM83A
shNC

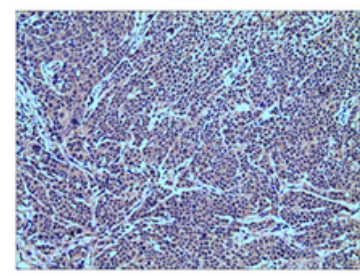

$100 \times$
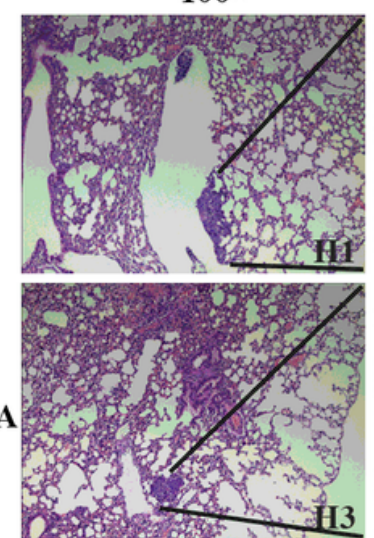

ShFAM83A
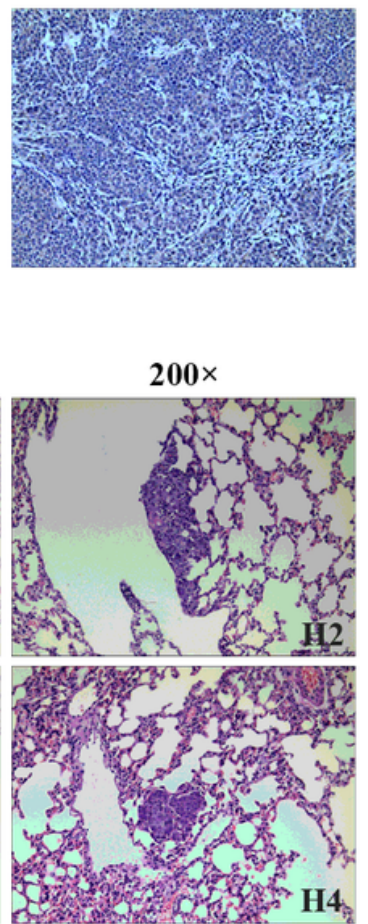


\section{Figure 6}

FAM83A knockdown inhibits HNSCC growth and metastasis in nude mice. A. The shFAM83A cells and control cells were subcutaneously injected into nude mice and general observation of tumor-bearing nude mice injected with cells. B. Volumes of xenograft tumors in shNC and shFAM83A groups. C. Tumor tissues in shNC and shFAM83A groups were weighed after 25 days. D. Representative showings of tumors formed in the nude mice were diagnosed as HNSCC by HE staining (100x). E. Representative photomicrographs of positive staining of FAM83A in shNC group and negative staining of FAM83A in shFAM83A group (100x). F. G. Representative photographs of lung tissues in mice injected with shFAM83A cells and shNC cells and a summary of the number of lung metastatic nodules in shFAM83A and shNC groups. $\mathrm{H}$. HE staining of lung tissues in shNC and shFAM83A groups $(\mathrm{H} 1, \mathrm{H} 3,100 \times ; \mathrm{H} 2, \mathrm{H} 4$, 200x). Data were statistically analyzed with Student's $t$-test and values are shown as the mean \pm SD. ${ }^{*}<<$ $0.05,{ }^{* \star} p<0.01,{ }^{* \star *} \mathrm{p}<0.001$. 
A

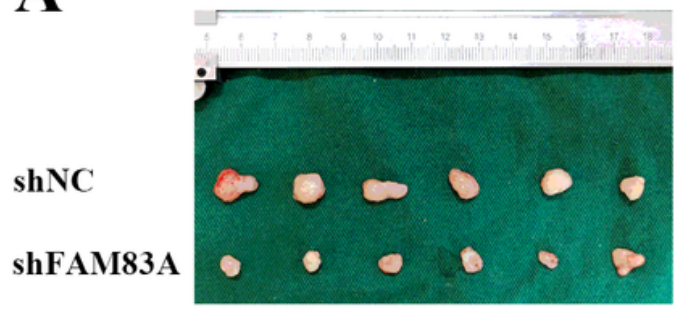

B

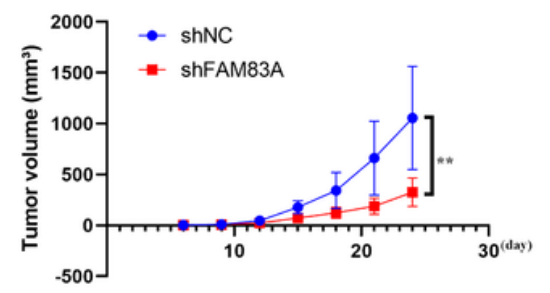

C

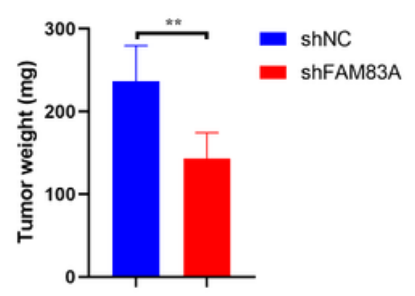

D
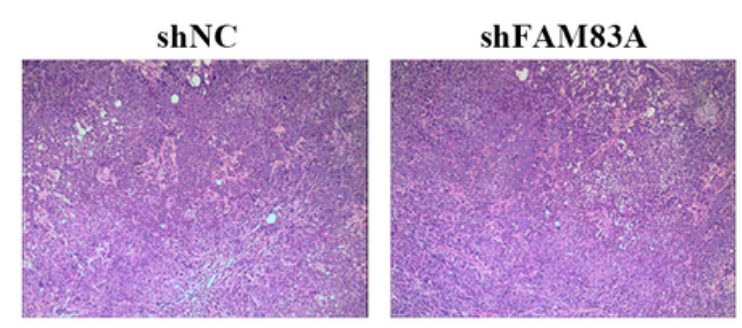

$\mathbf{F}$

shNC

shFAM83A

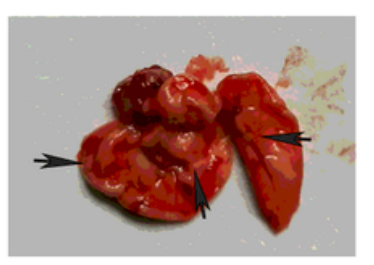

\section{G}

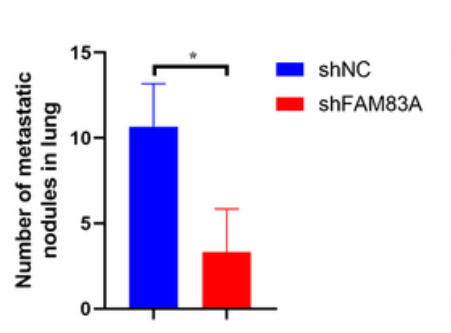

E
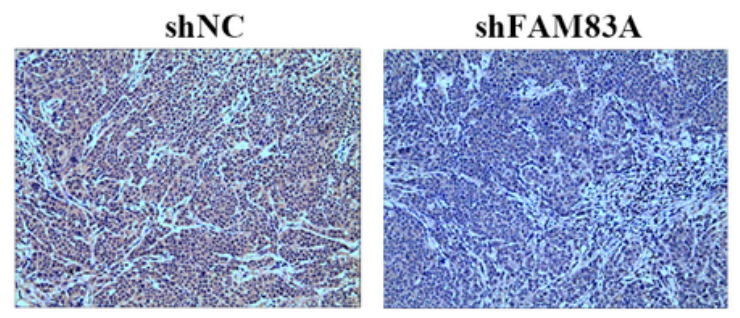

H

$\operatorname{shNC}$

shFAM83A
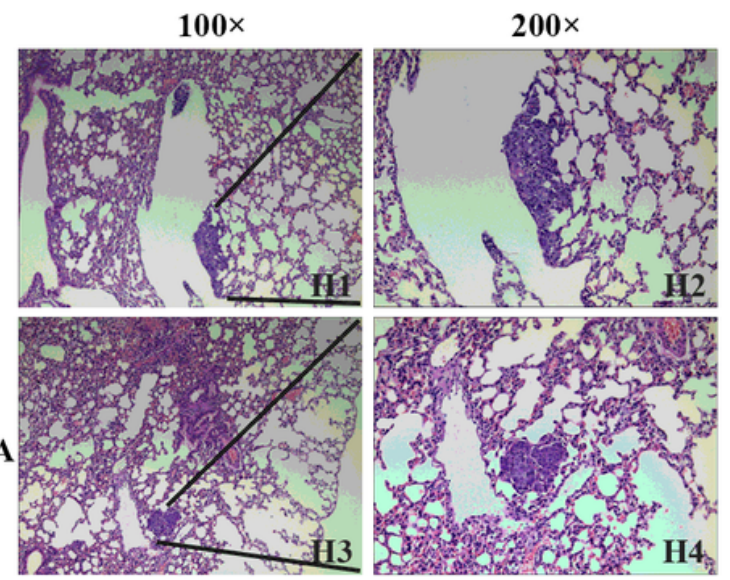

\section{Figure 6}

FAM83A knockdown inhibits HNSCC growth and metastasis in nude mice. A. The shFAM83A cells and control cells were subcutaneously injected into nude mice and general observation of tumor-bearing nude mice injected with cells. B. Volumes of xenograft tumors in shNC and shFAM83A groups. C. Tumor tissues in shNC and shFAM83A groups were weighed after 25 days. D. Representative showings of tumors formed in the nude mice were diagnosed as HNSCC by HE staining (100x). E. Representative photomicrographs of positive staining of FAM83A in shNC group and negative staining of FAM83A in 
shFAM83A group (100x). F. G. Representative photographs of lung tissues in mice injected with shFAM83A cells and shNC cells and a summary of the number of lung metastatic nodules in shFAM83A and shNC groups. $\mathrm{H}$. HE staining of lung tissues in shNC and shFAM83A groups $(\mathrm{H} 1, \mathrm{H} 3,100 \times ; H 2, H 4$, 200x). Data were statistically analyzed with Student's t-test and values are shown as the mean $\pm \mathrm{SD}$. ${ }^{*} \mathrm{p}<$ $0.05, * \star p<0.01, * * * p<0.001$. 